\title{
Difficulty in Formation of Counter-orbiting Hot Jupiters from Near-coplanar Hierarchical Triple Systems: A Sub-stellar Perturber
}

\author{
Yuxin $\mathrm{Xue}^{1}$ and Yasushi Suto ${ }^{1,2}$ \\ ${ }^{1}$ Department of Physics, The University of Tokyo, Tokyo 113-0033, Japan \\ 2 Research Center for the Early Universe, School of Science, The University of Tokyo, \\ Tokyo 113-0033, Japan \\ yuxin@utap.phys.s.u-tokyo.ac.jp
}

\begin{abstract}
Among a hundred transiting planets with a measured projected spin-orbit angle $\lambda$, several systems are suggested to be counter-orbiting. While they may be due to the projection effect, the mechanism to produce a counter-orbiting planet is not established. A promising scenario for the counter-orbiting planets is the extreme eccentricity evolution in near-coplanar hierarchical triple systems with eccentric inner and outer orbits. We examine this scenario in detail by performing a series of systematic numerical simulations, and consider the possibility of forming hot Jupiters, especially counterorbiting one under this mechanism with a distant sub-stellar perturber. We incorporate quadrupole and octupole secular gravitational interaction between the two orbits, and also short-range forces (correction for general relativity, star and inner planetary tide and rotational distortion) simultaneously. We find that most of systems are tidally disrupted and that a small fraction of survived planets turns out to be prograde. The formation of counter-orbiting hot Jupiters in this scenario is possible only in a very restricted parameter region, and thus very unlikely in practice.
\end{abstract}

Subject headings: planets and satellites: general - planets and satellites: formation planet-star interactions

\section{Introduction}

Ever since the first discovery of an exoplanet, $51 \mathrm{Peg}$ b, more than 100 Hot Jupiters (HJs) with semi-major axis $<0.1$ AU have been detected around main-sequence stars. Nevertheless their origin remains as one of the important unsolved puzzles in this field. It is generally believed that such gas giants are unlikely to be formed in-situ, and instead, that they are first formed at large distance from the central star, most likely beyond the ice line, and then migrated significantly inward to the current orbits (but see, e.g., Boley (2015) and Batygin (2015) for different ideas).

The migration mechanisms are not yet established, but possible scenarios include (1) diskplanet interaction (e.g., Lin et al. 1996; Alibert et al. 2005), (2) planet-planet scattering (e.g., 
Rasio \& Ford 1996; Nagasawa et al. 2008; Nagasawa \& Ida 2011; Beauge \& Nesvorny 2012), (3) the Lidov-Kozai migration (e.g., Lidov 1962; Kozai 1962; Wu \& Murray 2003; Fabrycky \& Tremaine 2007; Naoz et al. 2011; Petrovich 2015a; Anderson et al. 2016), and (4) secular migration (Wu \& Lithwick 2011).

In reality, those different migration mechanisms may have contributed to the observed HJ population to some degree. Each mechanism often predicts different statistical distribution and correlations of the resulting orbital parameters of the planetary systems, and the relevant observations may provide a potential clue to distinguish different mechanisms. For example, disk-planet interaction would imply that gas giants smoothly migrate inward in a gaseous disk and thus the angle, $\psi$, between the stellar spin and planetary orbital axes would not significantly change from its initial value (most likely very close to zero, but it is possible that the spin axis of the central star is moderately misaligned with the normal vector of the primordial disk (e.g., Bate et al. 2010; Foucart \& Lai 2011; Batygin 2012; Lai 2014). In contrast, the other migration mechanisms mentioned above rely on a dynamical process after the depletion of the gas disk, which can induce a strong spin-orbit misalignment. For this reason, measurement of $\psi$ can be a useful probe in understanding the origin of HJs.

Indeed, the Rossiter-McLaughlin effect has been very successful in measuring the sky projected spin-orbit angle, $\lambda$ for transiting planetary systems (Rossiter 1924; McLaughlin 1924; Queloz et al. 2000; Ohta et al. 2005; Winn et al. 2005). Approximately one-third of the measured systems exhibit significant misalignment of $\lambda>\pi / 4$, and a dozen of systems turned out to be even in a retrograde orbit $(\lambda>\pi / 2)$; see Fig.7 of Xue et al. (2014) for example. Such unexpected and counter-intuitive discoveries imply that those HJs should have experienced violent dynamical processes.

While all the above three dynamical migration mechanisms could produce retrograde HJs, it has been shown difficult to produce counter-orbiting HJs (e.g, Fabrycky \& Tremaine 2007; Naoz et al. 2011; Liu et al. 2015; Petrovich 2015b). For definiteness, we call the counter-orbiting planets as those with $160^{\circ}<\psi<180^{\circ}$, and retrograde planets are simply used to indicate $\psi>\pi / 2$ throughout the present paper even if the distinction may not be conventional.

In this context, we should note that the observed $\lambda$ differs from the true spin-orbit angle $\psi$; they are related in terms of the orbital inclination $i_{\text {orb }}$ and the obliquity of the stellar spin-axis $i_{\star}$ as

$$
\cos \psi=\cos i_{\star} \cos i_{\text {orb }}+\sin i_{\star} \sin i_{\text {orb }} \cos \lambda \approx \sin i_{\star} \cos \lambda .
$$

The above approximation holds for transiting systems with $i_{\mathrm{obs}} \approx \pi / 2$. Since the stellar axis is usually defined so that $0<i_{\star}<\pi / 2$, equation (1) implies that $\psi \geq \lambda$ if $0<\lambda<\pi / 2$ while $\psi \leq \lambda$ if $\pi / 2<\lambda<\pi$.

The true spin-orbit angle $\psi$ is not so easy to obtain, but can be estimated by combining the measurement of $i_{\star}$ via asteroseismology (Unno et al. 1989; Gizon 2003; Huber et al. 2013; Campante 2014; Christensen-Dalsgaard 2014). Benomar et al. (2014) performed the first quantitative determination of $\psi$ for transiting planetary systems around main-sequence stars. For HAT-P-7, their 
asteroseismology analysis yields $i_{\star} \approx 30^{\circ}$, and they obtain $\psi \approx 120^{\circ}$ from the joint analysis of the Rossiter-McLaughlin measurement of $\lambda \approx 180^{\circ}$. For Kepler-25c, they obtain $i_{\star}=65^{\circ} .4_{-6^{\circ} .4^{\circ}}^{+10^{\circ}}$, and $\psi=26^{\circ} .9_{-9^{\circ} .2}^{+7^{\circ} .0}$, which should be compared with $\lambda=9^{\circ} .4 \pm 7^{\circ} .1$. Indeed these results demonstrate the importance of the projection effect mentioned above. More importantly, planetary systems with $\lambda \approx 180^{\circ}$ may not be necessarily counter-orbiting, but just retrograde. This may also be the case for HAT-P-6b with $\lambda=165^{\circ} \pm 6^{\circ}$ (Albrecht et al. 2012), HAT-P-14b with $\lambda=189^{\circ} .1 \pm 5^{\circ} .1$ (Winn et al. 2011).

Therefore the existence of the counter-orbiting planets has not yet been established observationally so far. Nevertheless, it is tempting to consider a dynamical model that can theoretically explain the counter-orbiting HJs if exist at all. One promising mechanism has been recently proposed by Li et al. (2014). They consider a near-coplanar hierarchical triple system, and derived a flip condition that the inner planet becomes counter-orbiting under the secular perturbation up to the octupole-order of the gravitational potential of the outer object in a very eccentric orbit.

To be more specific, their flip condition is written as

$$
\epsilon>\epsilon_{\mathrm{crit}, \mathrm{i}}=\frac{8}{5} \frac{1-e_{1, i}^{2}}{7-e_{1, i}\left(4+3 e_{1, i}^{2}\right) \cos \left(\omega_{1, i}+\Omega_{1, i}\right)},
$$

in terms of

$$
\epsilon \equiv \frac{a_{1}}{a_{2}} \frac{e_{2}}{1-e_{2}^{2}}
$$

that characterizes the ratio of the orbit-averaged octupole to quadrupole terms in the massless limit $\left(m_{1} \ll m_{0}, m_{1} \ll m_{2}\right)$. In the above expressions, $e, a, \omega, \Omega$, and $m$ denote the eccentricity, semi-major axis, argument of periastron, longitude of ascending node with the subscripts 1 and 2 indicating the inner and outer body, respectively. In the massless limit, $a_{1}, a_{2}$, and $e_{2}$ are conserved, and thus $\epsilon$ defined by equation (3) is also a constant of motion. The other orbital elements are time-dependent, and we use the subscript $i$ in equation (2) in order to indicate their initial values.

Petrovich (2015b) presented a more general form of the flip condition (2) on the basis of the conservation of the energy (the orbit-averaged quadrupole and octupole potential terms) for the coplanar hierarchical triple configuration. His result, equation (11) of Petrovich (2015b), can be written as

$$
\epsilon_{\mathrm{L}}<\epsilon_{\mathrm{oct}} \equiv \frac{m_{0}-m_{1}}{m_{0}+m_{1}} \frac{a_{1}}{a_{2}} \frac{e_{2}}{1-e_{2}^{2}}<\epsilon_{\mathrm{U}},
$$

which reduces to equation (2) in the massless (or test-particle) limit of the inner planet. The lower and upper limits, $\epsilon_{\mathrm{L}}$ and $\epsilon_{\mathrm{U}}$, defining the boundary of the flip region are determined by the value of the final angle $\varpi_{f} \equiv \cos ^{-1} \hat{\mathbf{e}}_{1, f} \cdot \hat{\mathbf{e}}_{2, f}$ between the inner and outer orbital unit Lenz vectors. Specifically $\epsilon_{\mathrm{L}}$ and $\epsilon_{\mathrm{U}}$ correspond to $\varpi_{f}=0$ and $\pi$, respectively. The upper limit can be practically neglected for sub-stellar perturbers as considered in the present study, but is very important for planetary perturbers (Xue, Masuda, \& Suto, in preparation). 
Li et al. (2014) numerically computed the evolution of such coplanar triple systems in the massless limit, and confirmed that the flip condition is very well described by equation (2). Also, in the large inclination regime, that analytical flip criterion agrees well with the numerical results even up to $m_{2} / m_{1}>7$ (Teyssandier et al. 2013). They found that $e_{1}$ increases monotonically and the mutual orbital inclination between the two bodies, $i_{12}$, remains low just before the flip, and then the orbital flip of the inner planet proceeds in a very short timescale when $e_{1}$ becomes very close to unity, $1-e_{1} \sim 10^{-3}-10^{-4}$. In that case, the angular momentum of the inner planet is roughly given as $m_{1} \sqrt{G m_{0}\left(1-e_{1}^{2}\right)} \approx m_{1} \sqrt{2 G m_{0}\left(1-e_{1}\right)}$, and even a small perturbative torque may easily change the angular momentum of the inner planet, and thus flip its orbit if the value of $1-e_{1}$ is sufficiently small. Li et al. (2014) proposed a coplanar-flip mechanism for the formation of counter-orbiting HJs in which the inner planet flips by $\sim 180^{\circ}$ before the tidal evolution dominates, and then its extremely eccentric orbit is quickly circularized due to the strong tidal interaction by the central star.

Liu et al. (2015) found, however, that the short-range forces, General Relativity(GR), planetary tide (non-dissipative) and rotational distortion suppress the extreme value of $e_{1}$ that otherwise could be achieved due to the octupole term in hierarchical triple systems with large mutual orbital inclination (i.e., not coplanar configuration) in the Lidov-Kozai oscillation. These additional forces induce a precession of the Lenz vector of the inner planet, and impose a strict upper limit on the maximum achievable value of $e_{1}$; as the short-range forces become stronger, the orbital flips are more confined to the region where the mutual orbital inclination $i_{12}$ is close to $90^{\circ}$. This result strongly implies that one needs to incorporate those short-range forces in order to describe properly the dynamics of near-coplanar hierarchical triple systems, which is not taken into account in Li et al. (2014).

Petrovich (2015b) performed a series of such simulations for planetary perturbers including short-range force effects that induce pericenter precession of the inner orbit, such as GR, planetary tide and rotational distortion. All the resulting HJs in his simulations turn out to be in a prograde and low obliquity orbit. This is mainly because most of his initial conditions do not satisfy $\epsilon_{\mathrm{oct}}<\epsilon_{\mathrm{U}}$ in the flip condition (4) even when they satisfy $\epsilon_{\text {oct }}>\epsilon_{\mathrm{L}}$. Therefore his set of simulations does not cover the relevant parameter space for the formation of counter-orbiting HJs even though his simulations are for planetary perturbers, unlike for sub-stellar perturbers as we consider below.

Those interesting previous results motivated us to systematically explore the fate of the inner planet in near-coplanar hierarchical triple systems including quadrupole and octupole terms of the gravitational potential of the outer perturber, and short-range forces. Our simulation is based on the orbit-averaged secular dynamics following the formulation of Correia et al. (2011), in which the stellar and planetary spin effects are incorporated as well and the octupole order effect is included following Liu et al. (2015). Because we are primarily interested in the origin of counter-orbiting HJs, we consider only those systems that initially satisfy the analytical flip condition (2). The present paper focuses on the stellar perturber, and the parameter space relevant to the planetary perturber will be discussed in the next paper. In this case, we find that most of the systems are 
tidally disrupted and a fraction of survived planets remains mainly as prograde HJs; the formation of counter-orbiting HJs is possible only in a very restricted parameter range.

The rest of the paper is organized as follows. Section 2 describes the basic configuration of the hierarchical coplanar triple systems that we simulate. The simulation results are presented and discussed in $\S 3$ where we consider the parameter dependence in detail. Section 4 presents the spinorbit angle distribution in this scenario. Finally section 5 is devoted to summary and implications of the present paper. The set of equations that we employ is based on Correia et al. (2011) and Liu et al. (2015), but summarized explicitly in Appendix A for convenience and definiteness. The analytical expression for the short-range force effects are summarized in the Appendix B.

\section{Numerical Simulations}

An schematic configuration of near-coplanar hierarchical triple systems for our numerical simulations is illustrated in Figure 1. A central star of mass $m_{0}$ and radius $R_{0}$ is located at the origin of the coordinate. The normal vector of the invariable plane is the total orbital angular vector $\mathbf{G}_{\text {tot }}=\mathbf{G}_{1}+\mathbf{G}_{2}$ of the inner and outer bodies. Thus the mutual orbital inclination angle of the two orbits is given by $i_{12}=i_{1}+i_{2}$ where $i_{1}$ and $i_{2}$ are the inclinations of each orbit with respect to the invariable plane. Throughout the present paper, we adopt $m_{0}=1 M_{\odot}, R_{0}=1 R_{\odot}, m_{1}=1 M_{\mathrm{J}}$ and $R_{1}=1 R_{\mathrm{J}}$ for definiteness.

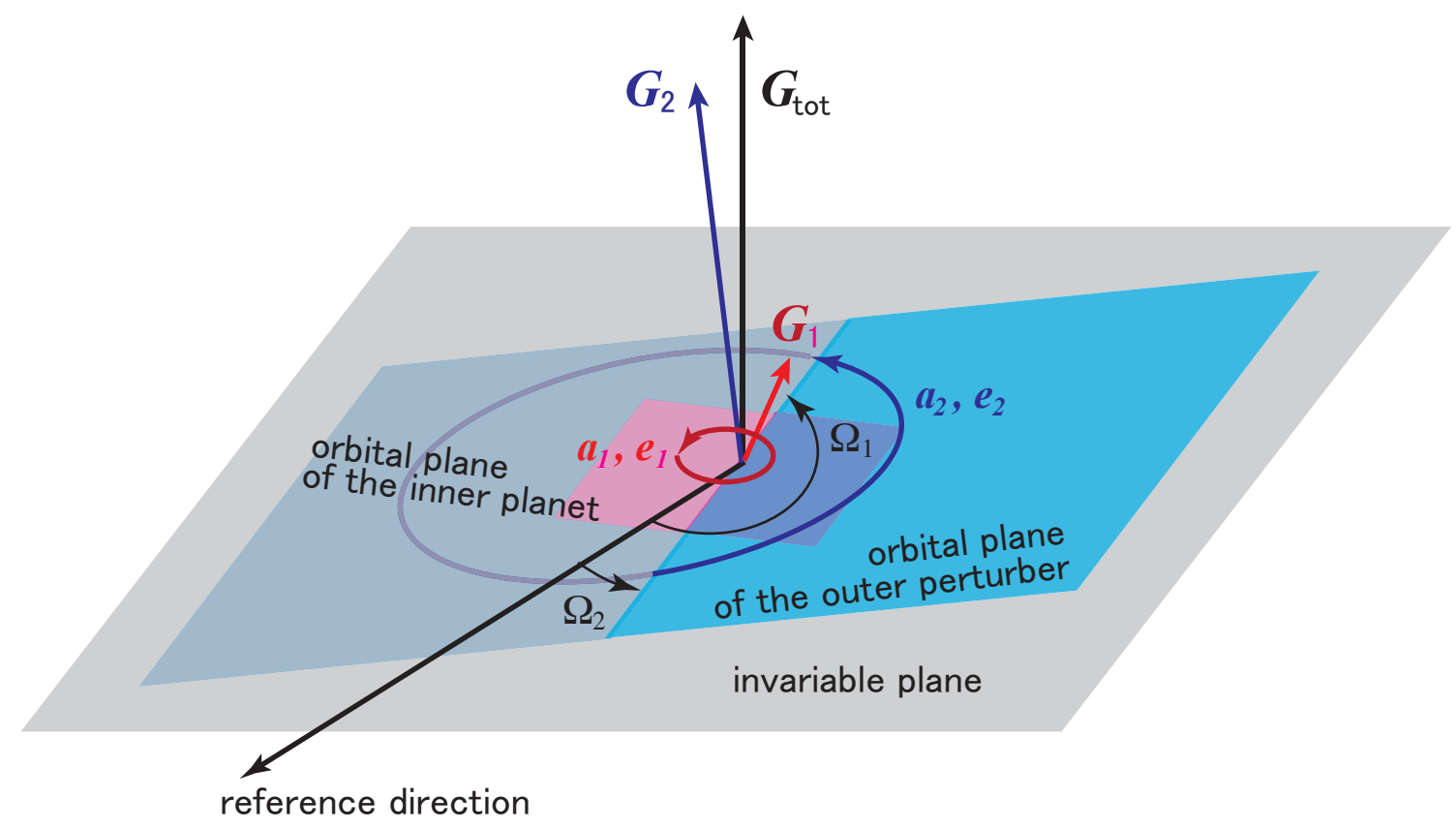

Fig. 1.- Schematic configuration of a near-coplanar triple system in the Jacobi coordinate. 
The equations of motions that we adopt are based on Correia et al. (2011), in which short-range forces, GR, spin rotation and tidal effects for both star and inner planet are included in addition to the quadrupole term of the orbit-average gravitation potential of the outer body. We modify their equations so as to incorporate the the octupole secular terms following Liu et al. (2015). The full equations of motion are described in Appendix A.

\begin{tabular}{c||c|c|c|c|c|c||c|c|c|c} 
model & $a_{2}(\mathrm{AU})$ & $e_{2}$ & $m_{2}\left(M_{\odot}\right)$ & $i_{12}$ & $t_{\mathrm{v}, \mathrm{p}}(\mathrm{yr})$ & $f$ & PHJ & RHJ & NM & $\mathrm{TD}$ \\
\hline fiducial & 500 & 0.6 & 0.03 & $6^{\circ}$ & 0.03 & 2.7 & $9.0 \%$ & $0.4 \%$ & $1.8 \%$ & $88.7 \%$ \\
\hline $\mathrm{m} 001$ & 500 & 0.6 & 0.01 & $6^{\circ}$ & 0.03 & 2.7 & $21.0 \%$ & $2.6 \%$ & $3.1 \%$ & $73.2 \%$ \\
$\mathrm{~m} 010$ & 500 & 0.6 & 0.1 & $6^{\circ}$ & 0.03 & 2.7 & $4.6 \%$ & $0.1 \%$ & $1.1 \%$ & $94.2 \%$ \\
$\mathrm{~m} 100$ & 500 & 0.6 & 1 & $6^{\circ}$ & 0.03 & 2.7 & $1.3 \%$ & $0.0 \%$ & $0.2 \%$ & $98.5 \%$ \\
\hline $\mathrm{a} 200$ & 200 & 0.6 & 0.03 & $6^{\circ}$ & 0.03 & 2.7 & $8.4 \%$ & $0.0 \%$ & $1.8 \%$ & $89.8 \%$ \\
$\mathrm{a} 100$ & 100 & 0.6 & 0.03 & $6^{\circ}$ & 0.03 & 2.7 & $7.7 \%$ & $0.0 \%$ & $1.1 \%$ & $91.2 \%$ \\
$\mathrm{a} 050$ & 50 & 0.6 & 0.03 & $6^{\circ}$ & 0.03 & 2.7 & $10.6 \%$ & $0.0 \%$ & $0.0 \%$ & $89.4 \%$ \\
\hline $\mathrm{e} 03$ & 500 & 0.3 & 0.03 & $6^{\circ}$ & 0.03 & 2.7 & $2.1 \%$ & $0.2 \%$ & $1.4 \%$ & $96.4 \%$ \\
$\mathrm{e} 04$ & 500 & 0.4 & 0.03 & $6^{\circ}$ & 0.03 & 2.7 & $3.7 \%$ & $0.2 \%$ & $0.7 \%$ & $95.4 \%$ \\
$\mathrm{e} 05$ & 500 & 0.5 & 0.03 & $6^{\circ}$ & 0.03 & 2.7 & $6.0 \%$ & $0.4 \%$ & $1.3 \%$ & $92.3 \%$ \\
$\mathrm{e} 07$ & 500 & 0.7 & 0.03 & $6^{\circ}$ & 0.03 & 2.7 & $13.5 \%$ & $0.5 \%$ & $3.1 \%$ & $82.9 \%$ \\
$\mathrm{e} 08$ & 500 & 0.8 & 0.03 & $6^{\circ}$ & 0.03 & 2.7 & $21.0 \%$ & $0.6 \%$ & $0.6 \%$ & $72.9 \%$ \\
\hline $\mathrm{i} 30$ & 500 & 0.6 & 0.03 & $30^{\circ}$ & 0.03 & 2.7 & $2.9 \%$ & $0.4 \%$ & $1.0 \%$ & $95.7 \%$ \\
$\mathrm{i} 15$ & 500 & 0.6 & 0.03 & $15^{\circ}$ & 0.03 & 2.7 & $4.6 \%$ & $0.7 \%$ & $1.5 \%$ & $93.1 \%$ \\
$\mathrm{i} 00$ & 500 & 0.6 & 0.03 & $0^{\circ}$ & 0.03 & 2.7 & $13.4 \%$ & $0.0 \%$ & $0.7 \%$ & $85.9 \%$ \\
\hline $\mathrm{t} 03000$ & 500 & 0.6 & 0.03 & $6^{\circ}$ & 0.3 & 2.7 & $3.4 \%$ & $0.0 \%$ & $2.2 \%$ & $94.4 \%$ \\
$\mathrm{t} 00030$ & 500 & 0.6 & 0.03 & $6^{\circ}$ & 0.003 & 2.7 & $55.3 \%$ & $25.3 \%$ & $1.8 \%$ & $17.6 \%$ \\
$\mathrm{t} 00003$ & 500 & 0.6 & 0.03 & $6^{\circ}$ & 0.0003 & 2.7 & $63.1 \%$ & $35.0 \%$ & $1.8 \%$ & $0.1 \%$ \\
\hline $\mathrm{f} 216$ & 500 & 0.6 & 0.03 & $6^{\circ}$ & 0.03 & 2.16 & $53.5 \%$ & $32.5 \%$ & $1.8 \%$ & $11.9 \%$ \\
$\mathrm{f} 166$ & 500 & 0.6 & 0.03 & $6^{\circ}$ & 0.03 & 1.66 & $60.6 \%$ & $37.6 \%$ & $1.8 \%$ & $0.0 \%$ \\
$\mathrm{f} 000$ & 500 & 0.6 & 0.03 & $6^{\circ}$ & 0.03 & 0.0 & $60.6 \%$ & $37.6 \%$ & $1.8 \%$ & $0.0 \%$ \\
\hline
\end{tabular}

Table 1: Summary of parameters and fates of our simulation runs. All the models adopt $m_{0}=1 M_{\odot}$, $R_{0}=1 R_{\odot}, m_{1}=1 M_{\mathrm{J}}, R_{1}=1 R_{\mathrm{J}}, \omega_{1, i}=0, \omega_{2, i}=0, \Omega_{1, i}=\pi, \Omega_{2, i}=0$, and $i_{s 1, i}=0$. The final states are divided into four categories: Prograde HJ (PHJ; $a<0.1 \mathrm{AU}, e<0.01$, and $i_{12}<\pi / 2$ ), Retrograde HJ (RHJ; $a<0.1 \mathrm{AU}, e<0.01$, and $i_{12}>\pi / 2$ ), Non-migrating planets (NM) and Tidally disrupted planets (TD; $q<R_{\text {roche }}$ ). We performed 1800 runs over the grids of $\epsilon_{i}-e_{1, i}$ plane for each model. 


\subsection{Model parameters}

In the present paper, we have in mind a sub-stellar object as the outer perturber. Specifically we adopt $a_{2, i}=500 \mathrm{AU}, m_{2}=0.03 M_{\odot}, e_{2, i}=0.6, i_{12, i}=6^{\circ}$, the viscous time scale for the inner planet, $t_{\mathrm{v}, \mathrm{p}}=0.03 \mathrm{yr}$, and $f=2.7$. The choice of those values for the fiducial parameters is admittedly rather arbitrary because it is very difficult to estimate their joint probability for actual near-coplanar hierarchical triples. Therefore we consider a variety of simulation models with fixed $m_{2}, a_{2, i}, e_{2, i}, i_{12, i}, t_{\mathrm{v}, \mathrm{p}}$, and $f$ as listed in Table 1 , instead of sampling those parameters from their assumed distribution function. Therefore our purpose is not to produce a mock distribution of real near-coplanar hierarchical triples, but to understand the parameter dependence of their dynamical evolution in a systematic fashion.

In each model, we perform 1800 different runs by varying $\left(e_{1, i}, \epsilon_{i}\right)$ systematically; $e_{1, i}$ is varied between 0.6 and 0.96 with a constant interval of 0.02 , and $\epsilon_{i}$ is varied between $\epsilon_{\text {crit,i }}$ and 0.15 with a constant interval of 0.001 . Thus the value of $a_{1, i}$ in each run is uniquely computed from $\epsilon_{i}$ through equation (2). We note that in all the models, both $a_{2}$ and $e_{2}$ are practically constant, i.e., $a_{2}=a_{2, i}$, and $e_{2}=e_{2, i}$, although $a_{1}$ and $e_{1}$ significantly change from their initial values in most cases.

We fix the initial spin periods of the central star and inner planet as 25day and 10day, the viscous time scale of the star $t_{\mathrm{v}, \mathrm{s}}$ as $50 \mathrm{yr}$, and the Love numbers for the star and inner planet as 0.028 and 0.5 , respectively. The dimensionless principal moment of inertia $I /\left(M R^{2}\right)$ of the star and inner planet are set to 0.08 and 0.26 , respectively. We do not randomly choose the initial phase angles so that $\epsilon_{\text {crit,i }}$ is independent of them in our parameter survey; we adopt $\omega_{1, i}=0, \omega_{2, i}=0$, $\Omega_{1, i}=\pi$, and $\Omega_{2, i}=0$. Since planets are generally expected to form within a protoplanetary disk that is perpendicular to the spin angular vector of the central star, the initial stellar inclination with respect to the orbit of the inner planet is set to $i_{s 1, i}=0$.

Following Petrovich (2015a), we divide the fate of the simulated systems into four different categories, and stop the run when it reaches one of the following states:

(i) PHJ (prograde HJ) : $a_{1, f}<0.1 \mathrm{AU}, e_{1, f}<0.01$ and $i_{12, f}<\pi / 2$.

(ii) RHJ (retrograde HJ) : $a_{1, f}<0.1 \mathrm{AU}, e_{1, f}<0.01$ and $i_{12, f}>\pi / 2$.

(iii) TD (tidally disrupted within the Roche limit of the central star) : The inner planet is tidally disrupted if its pericenter distance $q_{1} \equiv a_{1}\left(1-e_{1}\right)$ is less than the Roche limit:

$$
q_{1}<R_{\text {roche }} \equiv f\left(m_{0} / m_{1}\right)^{1 / 3}=0.0126\left(\frac{f}{2.7}\right) \mathrm{AU} \sim 2.71\left(\frac{f}{2.7}\right) R_{\odot} .
$$

The appropriate value for the Roche limit is somewhat uncertain. Thus while our fiducial value of $f$ is 2.7 (e.g, Guillochon et al. 2011), we consider $f=2.16$ (Faber et al. 2005) and $f=1.66$ (Naoz et al. 2012) as well. Note, however, that $f \approx 1$ corresponds to the radius of the central star itself, and the planet infalls to the star for $f<1$. 
(iv) NM (non-migrating planet) If the inner planet does not experience a significant migration, and stays at an orbit with $a_{1, f} \sim a_{1, i}$ until $t=10^{10} \mathrm{yr}$.

In the near-coplanar hierarchical triple systems as considered here, all the survived PHJs and RHJs turn out to be very well aligned $\left(i_{12}<10^{\circ}\right)$ and counter-orbiting HJs $\left(i_{12} \sim \pi\right)$, respectively.

Table 1 summarizes the model parameters of our simulations as well as the fraction of their final states. We should emphasize here that the fraction of the final states listed in Table 1 is computed assuming the uniform distribution over the surveyed region of $e_{1, i}-\epsilon_{i}$ plane. In reality, it is unlikely that $e_{1, i}$ and $\epsilon_{i}$ (or equivalently $a_{1, i}$ ) are distributed uniformly. Nevertheless this is inevitable because we do not assume any model-dependent and very uncertain prior distribution function for $e_{1, i}$ and $\epsilon_{i}$ in this paper. Therefore the values of fraction referred to throughout the present paper needs to be interpreted with caution, but still provide an important measure of the fate of the systems.

\subsection{Fiducial case}

Figure 2 plots the final states of the inner planet in our fiducial model for coplanar hierarchical triple systems. In this particular example, we first explore the range of $0.005<\epsilon_{i}<0.15$ so as to make sure of the validity of the analytical flip conditions, equation 2 by Li et al. (2014) and equation 4 by Petrovich (2015b). Figure 2 clearly shows that the region below those flip conditions agree with that of non-migrating planets in our runs. So their conditions are accurate in distinguishing the significant migration and non-migration boundary, even if they do not necessarily lead to RHJs; see discussion below. Its most important conclusion is that retrograde HJs are very difficult to form, despite the fact that the plotted region of $e_{1, i}-\epsilon_{i}$ is chosen so as to satisfy the flip condition (2) in the massless limit; $\sim 90 \%$ of the systems are tidally disrupted, and $\sim 10 \%$ survive as prograde HJs. The fraction of retrograde HJs turn out to be less than 1\%. Since this may be a rather unexpected result, we plot the dynamical evolution of typical systems for $e_{1, i}=0.9$ in six panels of Figure 3 . While we adopt 10 days as the spin rotation period of the inner planet throughout the current analysis, it may be more relevant to use 10 hours as in the case of Jupiter. In reality, however, the result turns out to be fairly insensitive to the value as shown in Appendix $\mathrm{C}$ below.

The lower-limit of the analytical flip condition (2) by Li et al. (2014), $\epsilon_{\text {crit,i }}$ is a very good approximation for the necessary condition, but obviously not a sufficient condition because it is derived on the basis of orbital dynamics without short-range forces effects. Our simulation shows that $\epsilon_{\text {crit,i }}$ becomes slightly larger, especially for large $e_{1, i}$ (small $\left.a_{1, i}\right)$. The detail of short-range forces effects is described in section 2.3. One example of the behavior in the region between the $\epsilon_{\text {crit,i }}$ we adopted and the real flip boundary including the short-range forces effects for $\epsilon_{i}=0.025\left(a_{1, i}=\right.$ 13.21AU) is illustrated in Figure 3a. The system exhibits an oscillation both in $1-e_{1}$ and $i_{12}$, but the resulting pericenter distance $q_{1}$ is not small enough for the tidal effect to operate. Thus the semi-major axis $a_{1}$ stays constant, and no significant migration occurs for $10^{10} \mathrm{yrs}$. All the other 


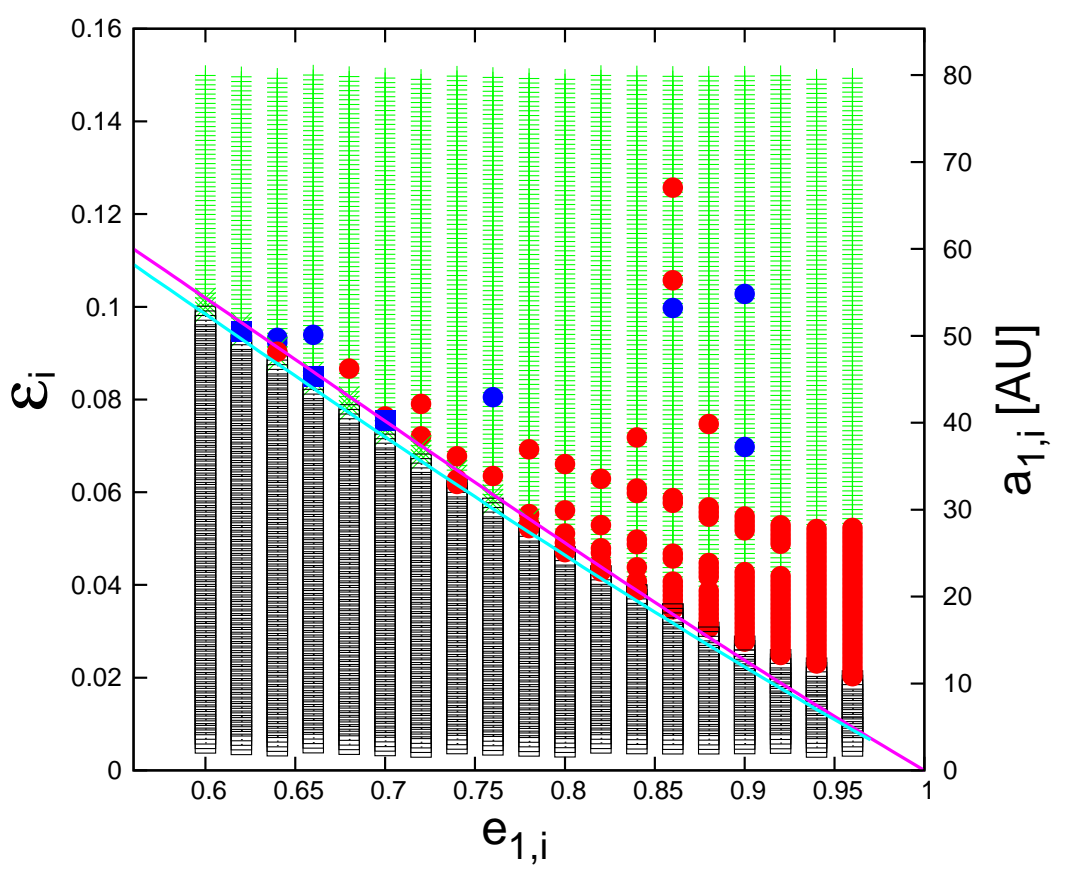

fiducial model

$\mathrm{m}_{2}=0.03 \mathrm{M}_{\odot}$

$a_{2, i}=500 \mathrm{AU}$

$\mathrm{e}_{2, \mathrm{i}}=0.6$

$\mathrm{i}_{12, \mathrm{i}}=6^{\circ}$

$\mathrm{t}_{\mathrm{v}, \mathrm{p}}=0.03 \mathrm{yr}$

$\mathrm{f}=2.7$

TD

NM

- $\mathrm{PHJ}$

- RHJ

- Li et al. (2014)

Petrovich (2015b)

Fig. 2.- Fate of the inner planet on the $e_{1, i}-\epsilon_{1, i}$ plane for our fiducial model; $a_{2, i}=500 \mathrm{AU}$, $m_{2}=0.03 M_{\odot}, e_{2, i}=0.6, t_{\mathrm{v}, \mathrm{p}}=0.03 \mathrm{yr}$. The values of $e_{1, i}$ are chosen from 0.6 to 0.96 with a constant interval of 0.02 , and $\epsilon_{i}$, from 0.005 to 0.15 with a constant interval of 0.001 . The final states are indicated by green crosses for Disrupted planets (TD), black open squares for Nonmigrating planets (NM), red filled circles for Prograde Hot Jupiters (PHJ), and blue filled circles for Retrograde Hot Jupiters(RHJ), respectively.

systems with $\epsilon_{i}<\epsilon_{\text {crit,i, }}$, therefore not simulated in the present paper, show the same behavior.

If $\epsilon_{i}$ is slightly larger than $\epsilon_{L}$, the amplitude of oscillation in $1-e_{1}$ becomes larger as plotted in Figure 3b for $\epsilon_{i}=0.028\left(a_{1, i}=14.81 \mathrm{AU}\right)$. In this case, the maximum eccentricity reaches 0.998, rather than 0.990 in the non-migrating example. This large-amplitude oscillations allow the inner planet reaching a minimum pericenter distance of $a_{1}\left(1-e_{1}\right) \sim 0.03 \mathrm{AU}$ where tidal dissipation efficiently extracts orbital energy, which results in gradual damping of $a_{1}$ at each maximum eccentricity (minimum pericenter distance). Thus PHJ systems form via multiple close approaches within a typical timescale of several $10^{9} \mathrm{yr}$. The example of Figure $3 \mathrm{~b}$ results in a HJ at $a \sim 0.065$ AU with $i_{12} \sim 4^{\circ}$. Indeed this slow coplanar migration is systematically studied by Petrovich (2015b), who proposes this as a potential path to PHJs, and our results are in agreement with his proposal.

As $\epsilon_{i}$ increases further, the octupole potential starts to dominate and drives $e_{1}$ very close to unity. At the same time, the orbit flip happens if the dissipative tide is neglected. Along the line of $e_{1}=0.9$, we observe two continuous regions where PHJs form (9.0\%) via the coplanar-flip 
mechanism. One example in this region is shown in Figure $3 \mathrm{c}$ for $\epsilon_{i}=0.034\left(a_{1, i}=18.01 \mathrm{AU}\right)$. Its suggests that this path to PHJ happens over a much smaller time-scale than that of Figure $3 \mathrm{~b}$; note the different scales of time in each panel. In this case, $1-e_{1}$ monotonically decreases and becomes close to $\sim 10^{-3}$ where the tidal effect becomes important. Therefore in the middle of increasing $i_{12}$, the system starts to be circularized and becomes PHJ with $a_{1 . f} \sim 0.035$ AU within $<10^{7}$ yrs. The mutual orbital inclination oscillates with gradually increasingly the amplitude in the range of $i_{12} \sim 0^{\circ}-30^{\circ}$, and then damps from $\sim 22^{\circ}$ to $\sim 9^{\circ}$ during the circularization stage. Since the eccentricity increases until the end of orbit flip if no short-range force effects are taken into account, such HJs have relatively low $i_{12}$. In total, the resulting PHJs (PHJ 9.0\%) are preferentially located in the low $\epsilon$ region. Most of them are formed through the coplanar-flip mechanism within a very short timescale $\left(\sim 10^{7} \mathrm{yr}\right)$, while a few result from secular tidal damping via eccentricity-inclination oscillation.

Beyond that value of $\epsilon_{i}$, the orbit of the inner planet is indeed flipped, but the fate changes very sensitively due to the subtle competition between the flipped condition and the tidal disruption as illustrated in Figure 3d to f. As a result, the system behavior looks chaotic, and there seems no systematic parameter region for the formation of RHJ (see Figure 2).

Evolution for the formation of RHJ similar to Figure 3d and e happens only in a very narrow parameter range; $\epsilon_{i}=0.070\left(a_{1, i}=37.21 \mathrm{AU}\right)$ and $\epsilon_{i}=0.103\left(a_{1, i}=54.81 \mathrm{AU}\right)$, respectively. The former is circularized at the second closest point of $1-e_{1}$. The orbit suffers from tidal circularization during an orbit flip process within a timescale of a few $10^{7}$ yr. Since the eccentricity of the inner orbit increases in the orbit flip stage, the system suffers from tidal circularization in the beginning of the orbit flip stage in order not to be tidally disrupted. Thus, this system ends with $i_{12, f}=162^{\circ}$, only slightly smaller than the highest $i_{12}$ ever reached, $177^{\circ}$. While the latter is circularized at the first closest point due to the stronger perturbation of the outer body. The tidal circularization starts when the orbit flip process is completed. Since the tidal circularization does not modify $i_{12}$ significantly, $i_{12, f}$ remains almost unchanged in the counter-orbiting regime, $172^{\circ}$ with $\pm 1^{\circ}$ oscillation. Such a high value of $i_{12}$ suggests that the counter orbiting HJ can be formed via the coplanar-flip mechanism, which supports the conclusion of Li et al. (2014).

Figure 3f presents an example of a tidally disrupted inner planet for $\epsilon_{i}=0.113\left(a_{1, i}=60.10 \mathrm{AU}\right)$. Its pericenter falls into the Roche limit at the second extreme eccentricity approach when $1-e_{1}$ reaches $\sim 2 \times 10^{-4}$. Such a state is preferentially found in systems in which the inner planet has a relatively large semi-major axis, since the gravitationally interaction between two orbits are stronger when the inner orbit reaches the extreme eccentricity. The comparison among the panels $\mathrm{d}$, e and $\mathrm{f}$ as well as Figure 2 strongly indicates that the fate of the systems is very sensitive to the parameters. Nevertheless the conclusion that most of the systems satisfying the flip condition (2) are tidally disrupted, instead of forming counter-orbiting HJs, is quite general. 

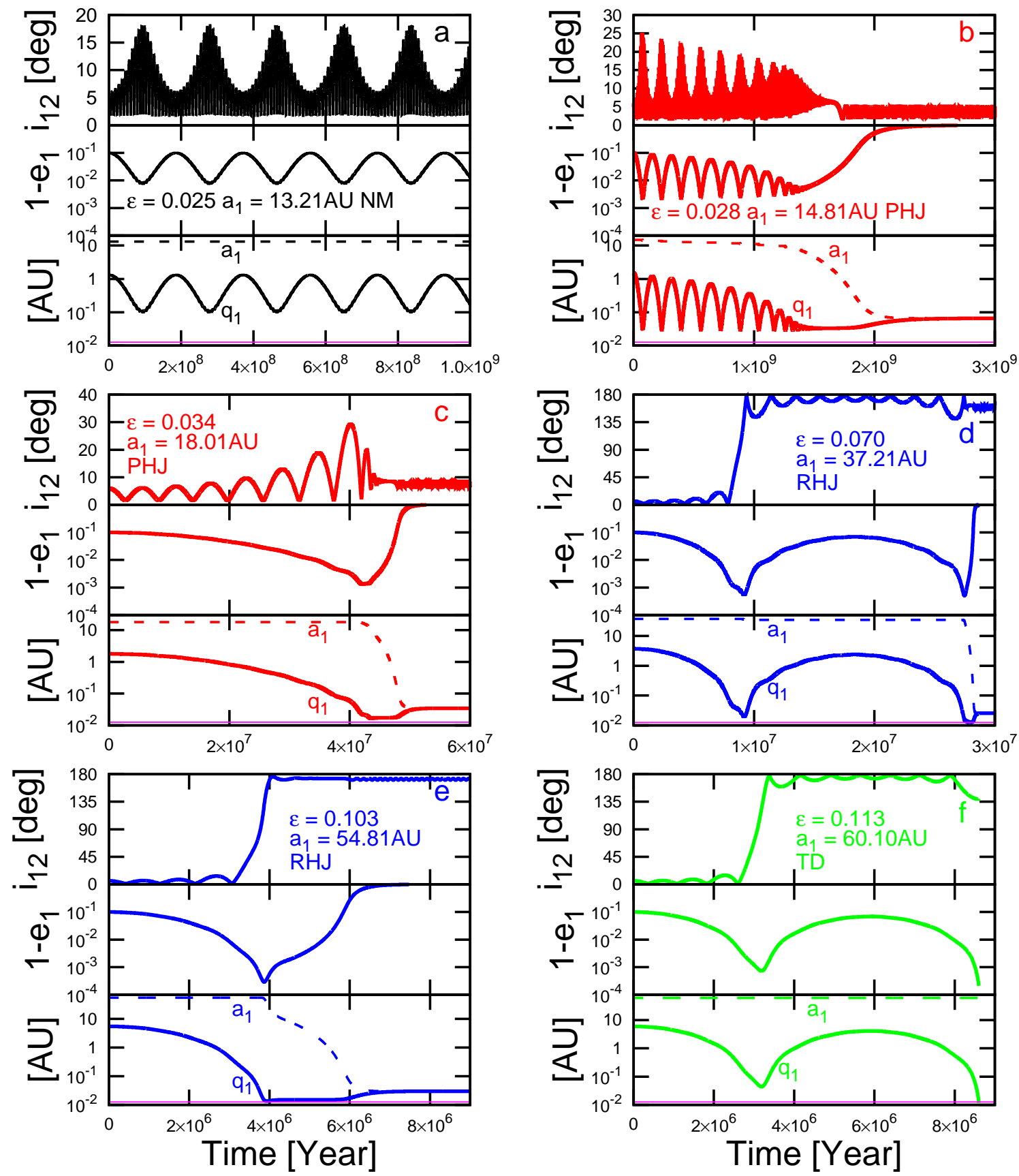

Fig. 3.- Evolution of our fiducial model with $e_{1, i}=0.9$ for different initial semi-major axis $a_{1, i}$. The final outcomes, Disrupted(TD), Non-migrating(NM), Prograde hot Jupiter(PHJ), and Retrograde hot Jupiter(RHJ) are shown in green, black, red, and blue line, respectively. For each time evolution, the evolution of $i_{12}, e_{1}$, and $a_{1}, q_{1}$ are shown in the top, middle and bottom panel, while $a_{1}$ is shown in dashed line, $i_{12}, e_{1}$, and $q_{1}$ are shown in solid line, and Roche limit is shown in the bottom panel with pink solid line, respectively. 
Since most of systems become disrupted via the orbital flip in our simulation, the condition of forming retrograde or counter-orbiting HJs is fairly fine-tuned. Considering the two successful examples of RHJs as shown above, a subtle change of initial condition may singnificantly modify the evolution and tidally disrupt the system as shown in Figure 2. So we may need to fine-tune the parameter sets in order to successfully make RHJs, which seems to be unlikely. Based on the low ratio (RHJ $0.4 \%$ ) and such an uncertainty, it is difficult to form retrograde or counter-orbiting HJs via the coplanar-flip mechanism.

Before moving to the next subsection, we would like to note that there is an interesting pattern in Figure 2; there are a few branching structures in prograde hot Jupiters. These are more significant in Figures 10 and 11 below. Although we are not yet successful in explaining the behavior, we suspect that they are related to some timescales in orbital evolution. We hope to come back to the issue in our next paper.

\subsection{Effect of short-range forces}

Liu et al. (2015) showed that the pericenter precessions due to short-range force effects suppress the growth of eccentricity of the inner planet, and reduce the flip region of $i_{12}$ for systems under the Lidov-Kozai oscillation. In this subsection, we show that the similar suppression works also for the near-coplanar triple systems.

A small area around the bottom-right region of Figure 2 corresponds to non-migrating planets despite the fact that they satisfy the flip criterion equation (2) initially. Indeed this comes from the short-range force effects. In order to see their effects separately, we consider the NM (non-migrating) planet example of Figure 3a $\left(\epsilon_{i}=0.025\left(a_{1, i}=13.21 \mathrm{AU}\right), e_{1, i}=0.9\right)$.

The left and right panels of Figure 4 plot the evolution of the mutual orbital inclination, $i_{12}$, and the pericenter distance of the inner planet in units of its initial semi-major axis. Since this example corresponds to the NM case, the latter is almost equivalent to $1-e_{1}$. We show the results for the secular orbital perturbation effect alone, orbital and general relativistic (GR) correction, orbital and planetary rotational distortion (PRD), orbital and planetary tide $(\mathrm{PT})^{1}$, and orbital and all the short-range force effects, from top to bottom.

As expected, the case without the short-ranges forces (top panels) flips the orbital inclination each time $1-e_{1}$ becomes less than $\sim 10^{-3}$. The flip repeats periodically since no other dissipational effects are included. If only the PRD is included, the system still shows the orbital flip, but the maximum value of $e_{1}$ is slightly suppressed relative to the purely orbital case.

\footnotetext{
${ }^{1}$ we include the central stellar tide and rotational distortion as well in our simulation, but their effects are indeed negligible.
} 

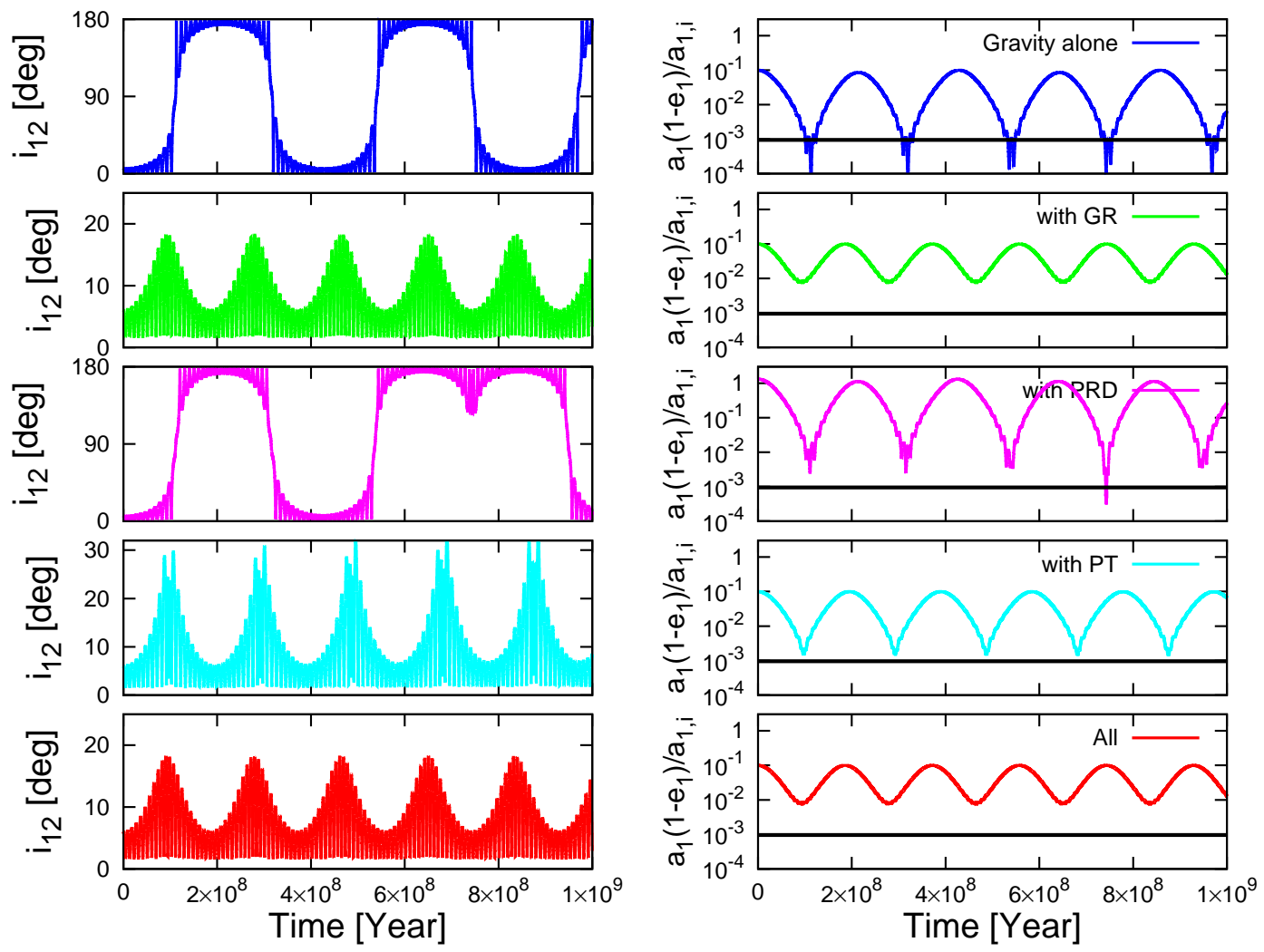

Fig. 4.- An illustrative example indicating the short-range force effects. The initial condition of this example corresponds to that of Figure 3a; $a_{1, i}=13.21 \mathrm{AU}\left(\epsilon_{i}=0.025\right)$, and $e_{1, i}=0.9$. Orbital evolution of $10^{9} \mathrm{yr}$ with different short-range force effects is plotted separately. From top to bottom, we plot quadrupole and octupole gravitational force alone in blue, gravity plus correction for general relativity (GR) in green, gravity plus planetary rotational distortion (PRD) in magenta, gravity plus tides (PT) in cyan, and finally gravity plus all the three short-range forces (All) in red. The black line corresponds to the Roche limit with $f=2.7$.

The precession due to PT could effectively limit the orbital flip with maximum eccentricity less than 0.999. On the other hand, the effect of GR is very effective in suppressing the eccentricity; the maximum value of $e_{1}$ under the GR correction barely reaches $\sim 0.99$. Thus the system stays outside the tidal circularization region for $10^{10} \mathrm{yr}$, and the PT never becomes important in reality, as shown in the bottom panels of Figure 4.

The above behavior can be understood by comparing the precession time-scales of the Lenz vector $\hat{\mathbf{e}}_{1}$ for those short-range forces, which we plot in Figure 5 on the basis of the expressions in Appendix B. Clearly the GR plays a dominant role for $e_{1}<0.995$, while PT becomes dominant for $e_{1}>0.995$; PRD is sub-dominant in either case. This is in good agreement with our simulation result shown in Figure 4, and therefore the precession induced by the short-range forces, in particular 


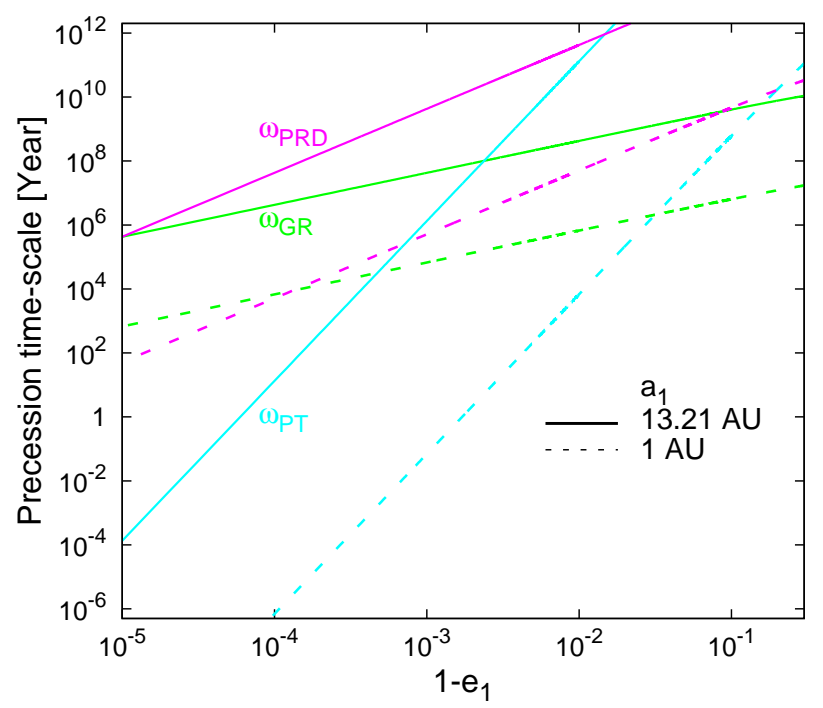

Fig. 5.- Analytical precession time-scales for the three short-range forces on $\hat{\mathbf{e}}_{1}$ as a function of $1-e_{1}$ (instead of $1-e_{1}^{2}$ ). The solid and dashed lines correspond to $a_{1}=13.21 \mathrm{AU}$ (corresponding to Figure 4) and $a_{1}=1 \mathrm{AU}$, respectively. The analytical expressions are explicitly given as equations $(\mathrm{B} .2) \sim(\mathrm{B} .4)$ in Appendix B.

GR, prevents the orbital flip. Since the short-range forces become stronger for the smaller semimajor axis, the NM planets are located around the high- $e_{1, i}$ and low- $\epsilon_{i}$ region.

\section{Dependence on the model parameters}

The previous section has presented the result for our fiducial model, and discussed the dynamical behavior for several examples. Next we consider the dependence of parameters employed in the fiducial model, separately in each subsection below. The full list of different models is summarized in Table 1, and we plot the two models in each subsection as examples. Since we already confirmed that planets with $\epsilon_{i}<\epsilon_{\text {crit }}$ do not migrate in practice, we run the models for $\epsilon_{\text {crit }}<\epsilon_{i}<0.15$ in what follows.

\subsection{Mass of the outer perturber}

We adopt $m_{2}=0.03 M_{\odot}$ as our fiducial value, but one might wonder if the larger mass would be more relevant as (sub-)stellar perturbers. While this sounds reasonable, the larger $m_{2}$ significantly increases the tidal disruption ratio, and there is no chance to form retrograde planets in practice.

This is clearly shown in the left and right panels of Figure 6 for $m_{2}=1 M_{\odot}$ and $0.1 M_{\odot}$, 

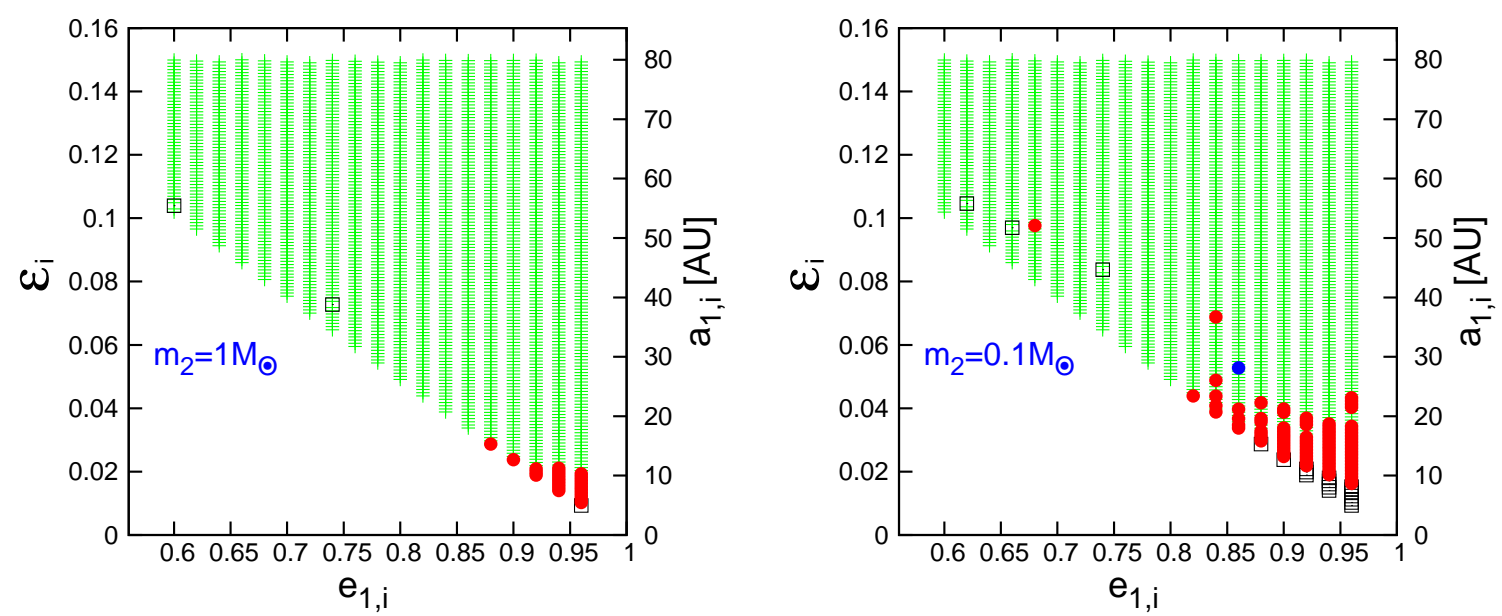

Fig. 6. - Final outcomes of m100 with $m_{2}=1 M_{\odot}$ (left) and m010 with $m_{2}=0.1 M_{\odot}$ (right) on $e_{1, i}-\epsilon_{i}$ plane.

respectively. Since we focus on the parameter space satisfying the analytic flip condition (2), the gravitational perturbation due to the outer body is sufficiently strong to produce the orbital flip potentially. Under such circumstances, the larger $m_{2}$ results in the larger $e_{1}$ (extremely closer to unity) in which leads to the stronger tidal effect. Therefore in order to survive the tidal disruption, the inner planet should have the smaller $a_{1, i}$ for the larger $m_{2}$. This is why the fractions of both PHJ and RHJ decreases as $m_{2}$ increases.

Thus it is very difficult to form RHJ via the near-coplanar flip mechanism if the outer perturber has a stellar mass $m_{2}>0.1 M_{\odot}$. This is why we adopt $m_{2}=0.03 M_{\odot}$ as our fiducial value.

\subsection{Semi-major axis of the outer perturber}

Consider next the dependence on $a_{2}$. Again the proper choice of this parameter is not easy. If $a_{2}$ is larger, the near-coplanar configuration is unlikely. On the other hand, the sub-stellar perturber closer to the central star may be difficult to form either. As a compromise, we select $a_{2}=500,200$, 100 and $50 \mathrm{AU}$ in Table 1 with $a_{2}=500 \mathrm{AU}$ being the fiducial value. Figure 7 presents the results for $a_{2}=200 \mathrm{AU}$ and 50AU. There are two important messages from Figure 7 .

First, RHJs do not form for $a_{2} \leq 200$ AU. In order to become a RHJ, the inner planet needs to experience the orbital flip before the tidal circularization. This prefers larger $a_{1, i}$ because the inner planet suffers from less tidal dissipation before reaching the extreme eccentricity for the orbital flip. Even larger $a_{1, i}$, however, results in stronger gravitational perturbation from the outer body, and thus the inner planet is tidally disrupted. Due to that subtle competition, RHJs in our fiducial model are confined in the narrow region of $0.07<\epsilon_{i}<0.11$. As $a_{2}$ decreases, the entire system 

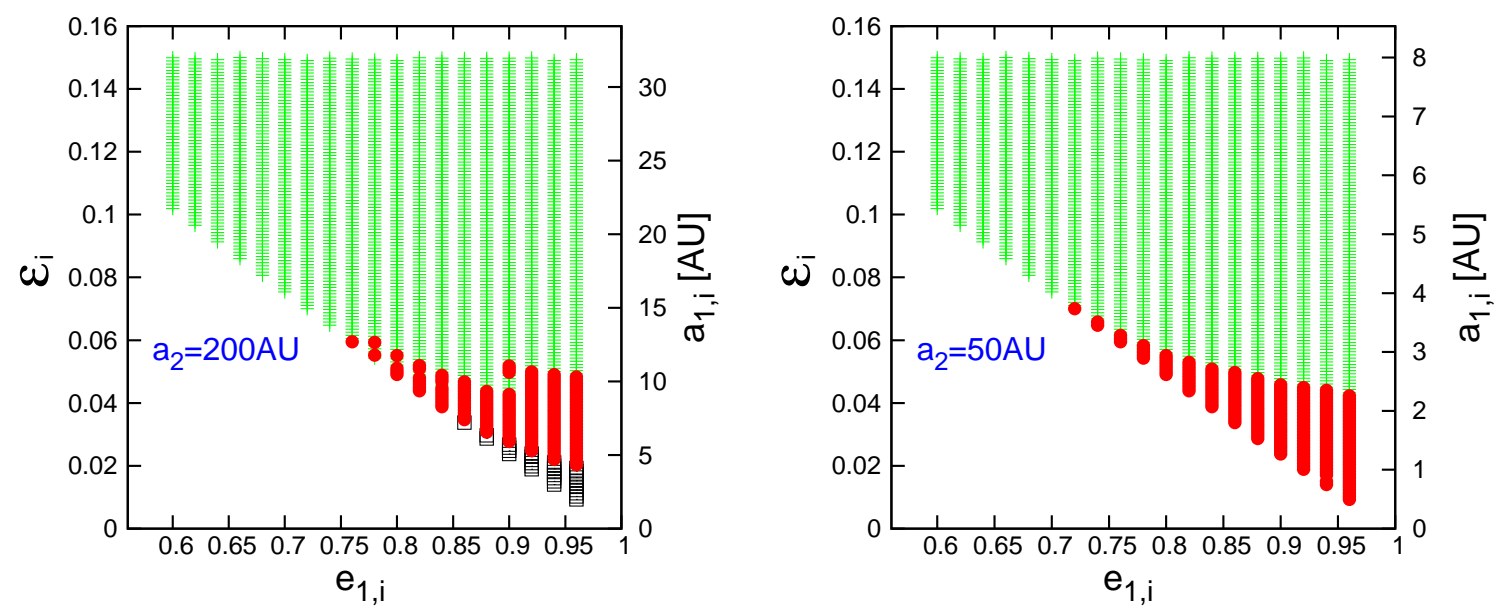

Fig. 7.- Final outcomes of a200 with $a_{2}=200 \mathrm{AU}$ (left) and a050 with $a_{2}=50 \mathrm{AU}$ (right) on $e_{1, i}-\epsilon_{i}$ plane.

becomes more compact for the same value of $\epsilon_{i}$. Thus the stronger gravitational perturbation of the outer body brings the inner planet to the orbit within the Roche limit more easily because the pericenter distance at the same maximum eccentricity is smaller. This is why RHJs disappear for the smaller $a_{2}$ models. For the same reason, PHJs are limited for the lower $\epsilon_{i}$ region.

Second, NM planet fraction drops as $a_{2}$ decreases; $1.8 \%, 1.8 \%, 1.1 \%$, and $0.0 \%$ for $a_{2}=$ 500,200,100, and $50 \mathrm{AU}$, respectively. In the fiducial model, short-range forces suppress the the maximum eccentricity and the inner planet does not flip nor is tidally circularized around the high$e_{1, i}$ and very low- $\epsilon_{i}$ region. The same value of $e_{i}$, however, corresponds to the smaller $a_{1}$ for the smaller $a_{2}$ models. Thus the pericenter distance for those systems becomes smaller, which enhances the tidal dissipation and thus circularizes the orbit. As a result, systems gradually migrate and finally become PHJs via the secular eccentricity-inclination oscillation as illustrated in Figure $3 \mathrm{~b}$. In any case, the formation of RHJs is more difficult for the smaller $a_{2}$ than the fiducial model.

\subsection{Eccentricity of the outer perturber}

Sub-stellar perturbers may exhibit a broad range of eccentricity, and we run six simulation sets with $e_{2, i}=0.3,0.4,0.5,0.6$ (fiducial), 0.7, and 0.8, and two examples out of those models are plotted in Figure 8. We find that the fraction of PHJs monotonically increases for the more eccentric outer perturber. PHJs tend to form preferentially in low $a_{1, i}$ where tidal dissipation becomes effective. Since we consider the same range of $\epsilon_{i}$ for all the models, the corresponding value of $a_{1, i}$ for the same $\epsilon_{i}$ becomes smaller as $e_{2, i}$ increases. Thus the dependence of the fraction on $e_{2}$ is mainly due to the scaling. While RHJs are very rare, their fraction also increases slightly as $e_{2}$, but it would be mainly due to the scaling of $a_{1, i}$ with respect to $\epsilon_{i}$. 

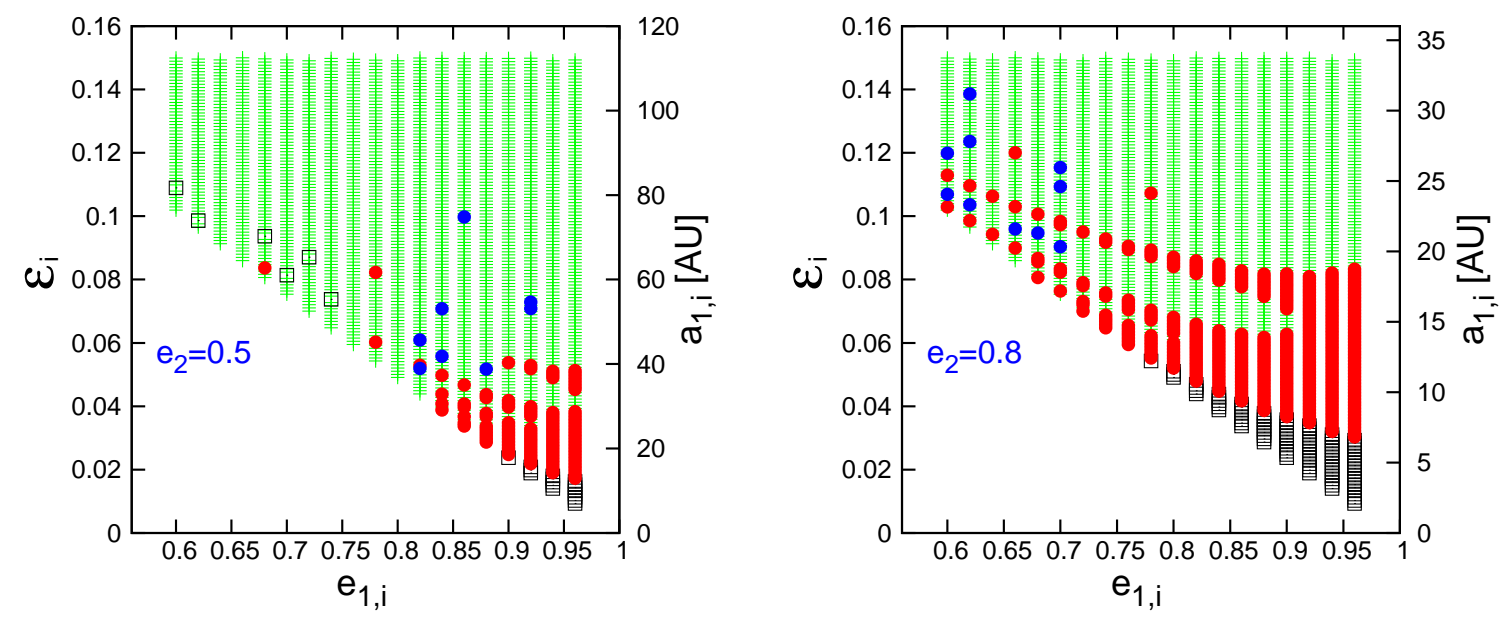

Fig. 8. - Final outcomes of e05 with $e_{2, i}=0.5$ (left) and e08 with $e_{2, i}=0.8$ (right) on $e_{1, i}-\epsilon_{i}$ plane.

\subsection{Mutual orbital inclination of the inner and outer orbits}

The initial orbits of the inner and outer bodies are naturally expected to be inclined to some extent. While our fiducial model adopts $i_{12, i}=6^{\circ}$, we examine more inclined cases of $i_{12, i}=15^{\circ}$ and $30^{\circ}$ as well as an idealized coplanar case $\left(i_{12, i}=0^{\circ}\right)$. The Lidov-Kozai mechanism starts to work for more inclined cases, and we do not consider here because the orbital flip does not happen in those cases as mentioned in Introduction.
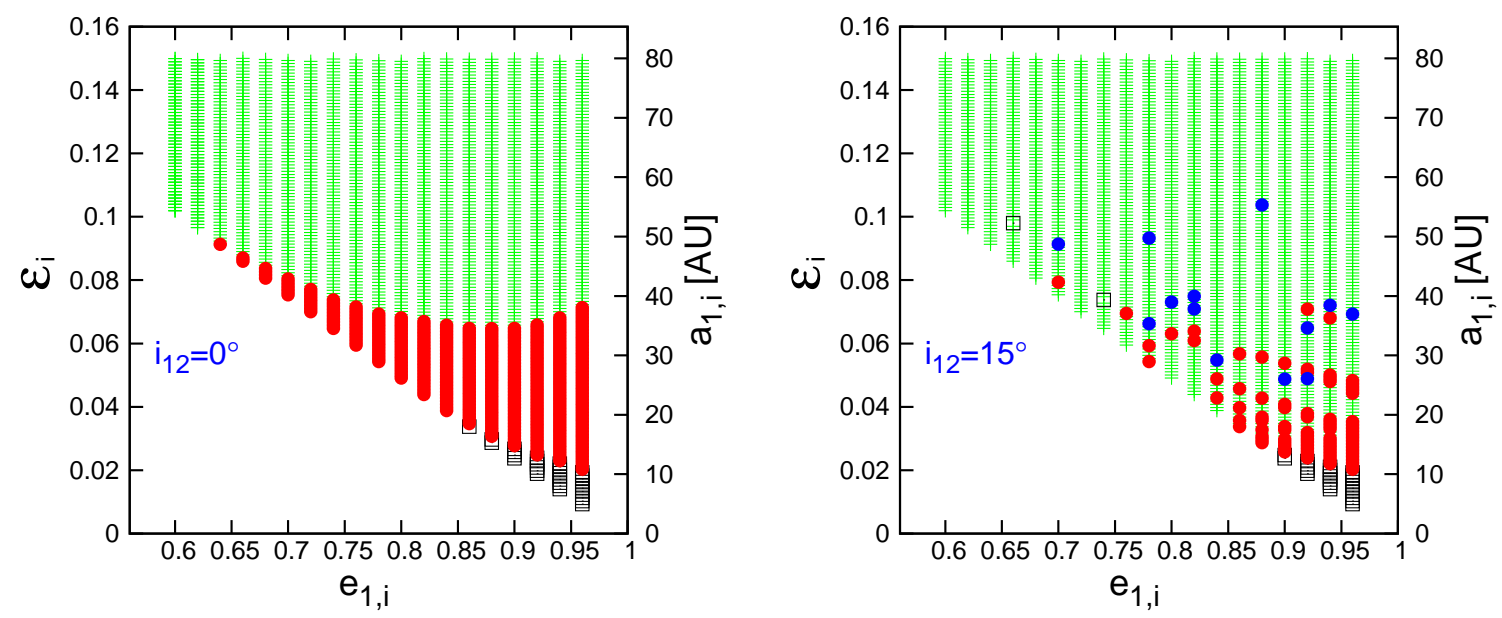

Fig. 9.- Final outcomes of i00 with $i_{12, i}=0$ (left) and i15 with $i_{12, i}=15^{\circ}$ (right) on $e_{1, i}-\epsilon_{i}$ plane. 
The left and right panels of Figure 9 present the results for $i_{12}=0$ and $i_{12}=15^{\circ}$. In the exact coplanar case, the net force normal to the orbital plane always vanishes, and the orbits cannot flip. Thus RHJs cannot form, but PHJs can.

As $i_{12, i}$ increases, the fraction of PHJs decreases monotonically, and they are confined around the narrow region with high- $e_{1, i}$ and low $\epsilon_{i}$. In rare cases, RHJs form in a scatter manner over the on $e_{1, i}-\epsilon_{i}$ plane, probably due to the chaotic nature of the system.

\subsection{Viscous time-scale of the inner planet}

Unfortunately it is well known that the viscous time-scale of planets, $t_{\mathrm{v}, \mathrm{p}}$, (equivalently, the tidal delay time and tidal quality factor) is the most uncertain parameter in the equilibrium tidal theory. The observational data for the Jupiter - Io system put an empirical lower limit on that of Jupiter as $t_{\mathrm{v}, \mathrm{J}}>15 \mathrm{yr}$. On the other hand, Socrates et al. (2012) stated that $t_{\mathrm{v}, \mathrm{p}}<1.5 \mathrm{yr}$ for an initially highly eccentric planetary orbit with semi-major axis of $\sim 5$ AU to be circularized into $<0.06 \mathrm{AU}$ within 10 Gyr. They argued that the discrepancy between their upper limit and the empirical lower limit for Jupiter should not be taken seriously given various theoretical uncertainties concerning the tidal dissipation model and diversities of the physical properties of the exoplanets.
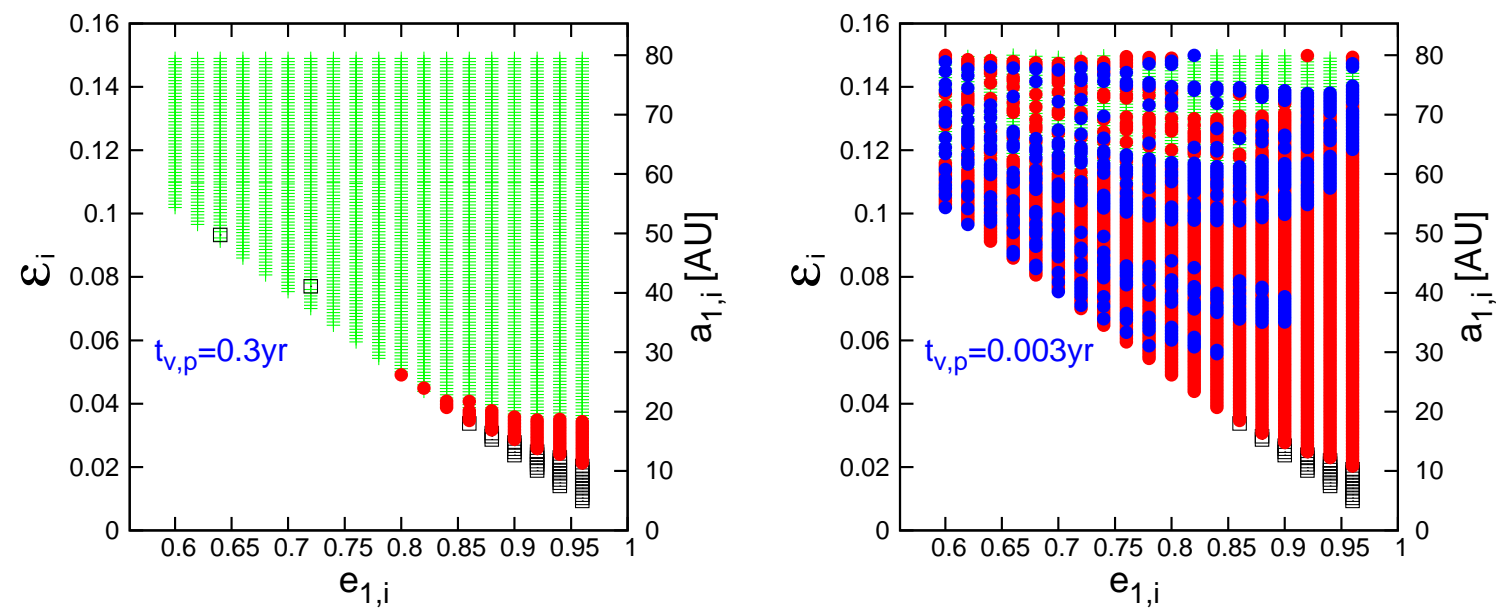

Fig. 10. - Final outcomes of t03000 with $t_{\mathrm{v}, \mathrm{p}}=0.3 \mathrm{yr}$ (left) and t00030 with $t_{\mathrm{v}, \mathrm{p}}=0.003 \mathrm{yr}$ (right) on $e_{1, i}-\epsilon_{i}$ plane.

For instance, more recent work by Storch \& Lai (2014) examined a possibility of tidal dissipation in solid cores of giant planets, and claimed that tidal dissipation in the core can reconcile the Jupiter-Io tidal constraint and very efficient high-eccentricity migration simultaneously.

Given a somewhat confusing situation, we decided to adopt $t_{\mathrm{v}, \mathrm{p}}=0.03$ as our fiducial value, simply following Li et al. (2014). Our purpose of the present paper is not to find a suitable value 
for $t_{\mathrm{v}, \mathrm{p}}$ but to understand the role of $t_{\mathrm{v}, \mathrm{p}}$ in the orbit flip of near-coplanar triple systems. Thus we examine the other three cases with $t_{\mathrm{v}, \mathrm{p}}=0.3,0.003$, and $0.0003 \mathrm{yr}$ as well.

The results are plotted in Figure 10. As expected, the fate of the inner planet is very sensitive to the very uncertain value of $t_{\mathrm{v}, \mathrm{p}}$. When $t_{\mathrm{v}, \mathrm{p}}$ is smaller, the tide on the planet becomes stronger and the planet suffers from very efficient circularization even at a larger pericenter distance. Thus the majority of the tidally disrupted planets for $t_{\mathrm{v}, \mathrm{p}}=0.3 \mathrm{yr}$ survive as PHJs and RHJs for $t_{\mathrm{v}, \mathrm{p}}=0.003 \mathrm{yr}$. The lower-right region of Figure 10 corresponds to planets at a relatively larger pericenter distance, and thus insensitive to the value of $t_{\mathrm{v}, \mathrm{p}}$.

Of course, the value of $t_{\mathrm{v}, \mathrm{p}}=0.003 \mathrm{yr}$ is very extreme and unrealistic; even the paucity of the observed RHJs is inconsistent with the choice. Nevertheless Figure 10 clearly illustrates that the uncertainty of the tidal dissipation model is the key to understanding the formation and dynamical evolution of HJs in general.

\subsection{The proportional constant for the Roche limit}

Finally we consider the criterion of the tidal disruption itself. As discussed in $\S 2.1$, the proportional factor $f$ of the Roche limit in equation (5) is not precisely determined. While we adopt $f=2.7$ following Guillochon et al. (2011) from hydrodynamical simulations, $f=2.16$ is reported by Faber et al. (2005) and $f=1.66$ is adopted in simulations by Naoz et al. (2012).
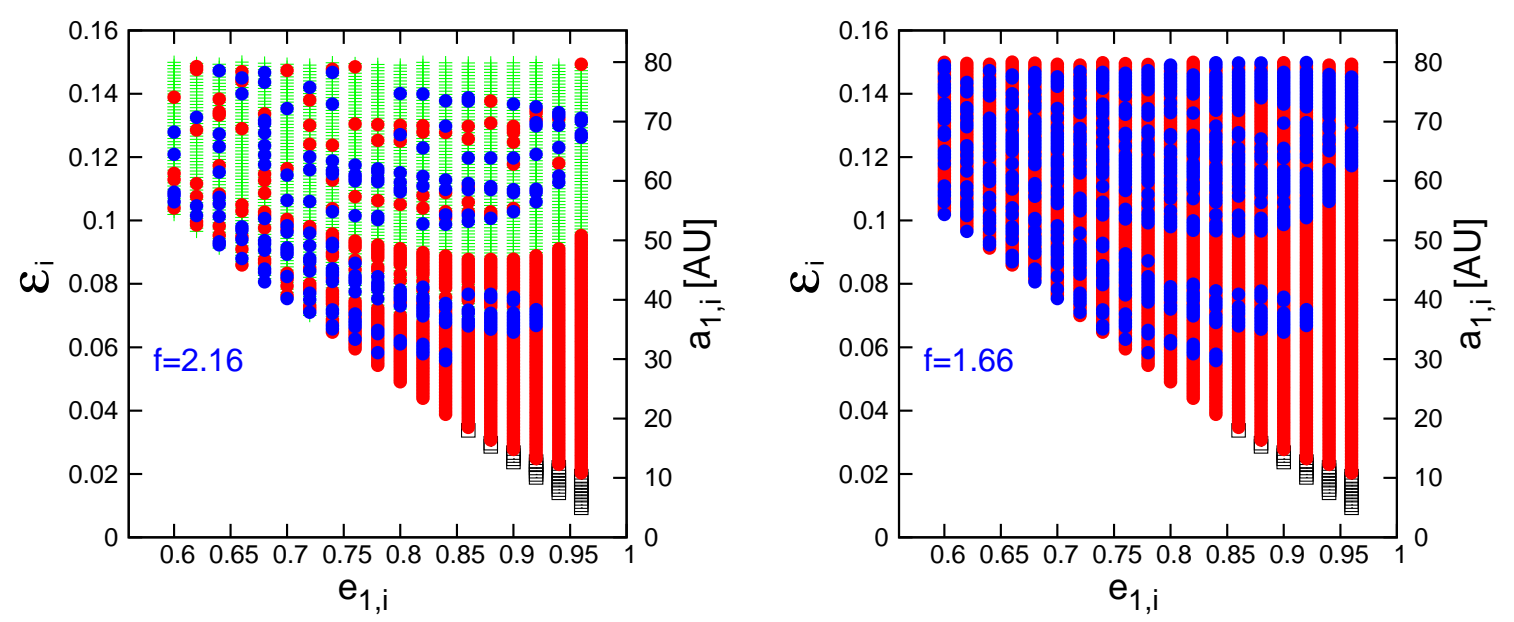

Fig. 11. - Final outcomes of f216 with $f=2.16$ (left) and f166 with $f=1.66$ (right) on $e_{1, i}-\epsilon_{i}$ plane.

As shown in the previous subsection, the efficiency of the tidal disruption is the most important in determining the fate of the inner planet. Thus we plot the cases of $f=2.16$ and $f=1.66$ in the left and right panels of Figure 11, respectively. 
Similarly to Figure 10, short-range forces are effective and suppress the growth of the eccentricity of the inner planet in the lower-right region of Figure 11. Thus the pericenter distance of the inner planets around the region is larger than $R_{\text {roche }}$ in any case, and the fudge factor $f$ hardly changes the evolution of those planets.

On the other hand, tidally disrupted planets in our fiducial model are sensitive to the value of $f$. As is clear from Figure 11, those planets turn out to survive as PHJs and RHJs for the smaller value of $f$, and there are no tidally disrupted planets for $f=1.66$. Indeed the result with $f=1.66$ is already virtually indistinguishable with the case where the tidal disruption happens only when the inner planet falls into the central star.

\section{Spin-orbit angle distribution}

So far we have classified the survived HJs into prograde or retrograde according to the mutual orbital inclination angle $i_{12}$ of the inner and outer orbits, i.e., $i_{12}<90^{\circ}$ or $>90^{\circ}$, respectively. In reality, however, $i_{12}$ cannot be measured directly since the possible outer perturbers of the observed HJs are hardly identified. Thus observationally the distinction between prograde and retrograde HJs is made from the the value of $\lambda$, the sky-projected angle of $i_{s 1}$, obtained from the RossiterMcLaughlin effect. Since our current simulation runs solve the evolution of the stellar spin axis as well, we can address the validity of a somewhat conventional assumption of $i_{12}=i_{s 1}$. The result is plotted in Figure 12, which basically confirms that $i_{12}$ can be used as a proxy for $i_{s 1}$ as long as the stellar spin vector is completely aligned with the orbital angular momentum vector of the inner planet initially $\left(i_{s 1, i}=0\right)$ as we adopted in the present runs.

Now we show the distribution of $i_{s 1}$ in Figure 13. These plots indicate that PHJs and RHJs in our simulations correspond almost exclusively to well-aligned $\left(i_{12}<20^{\circ}\right)$ and counter-orbiting $\left(180^{\circ}-i_{12}<20^{\circ}\right)$ planets. This is not the case, however, for models with very strong tidal interaction (t00030 and f216), which exhibit a very broad distribution of $i_{12}$ and thus of $i_{s 1}$.

In the coplanar-flip mechanism, the planetary orbit suffers from tidal circularization after the orbit flip. Thus the system ends up with PHJs if the tidal circularization happens before the orbit flip, and RHJs if the orbit flip occurs before the circularization. On the other hand, the tidally disrupted planets have a very broad distribution of $i_{s 1}$ that we define at the epoch when the pericenter distance of the inner planet reaches the Roche limit. The result implies that those planets fall into the Roche limit in a very short time-scale less than that of the orbit flip. 

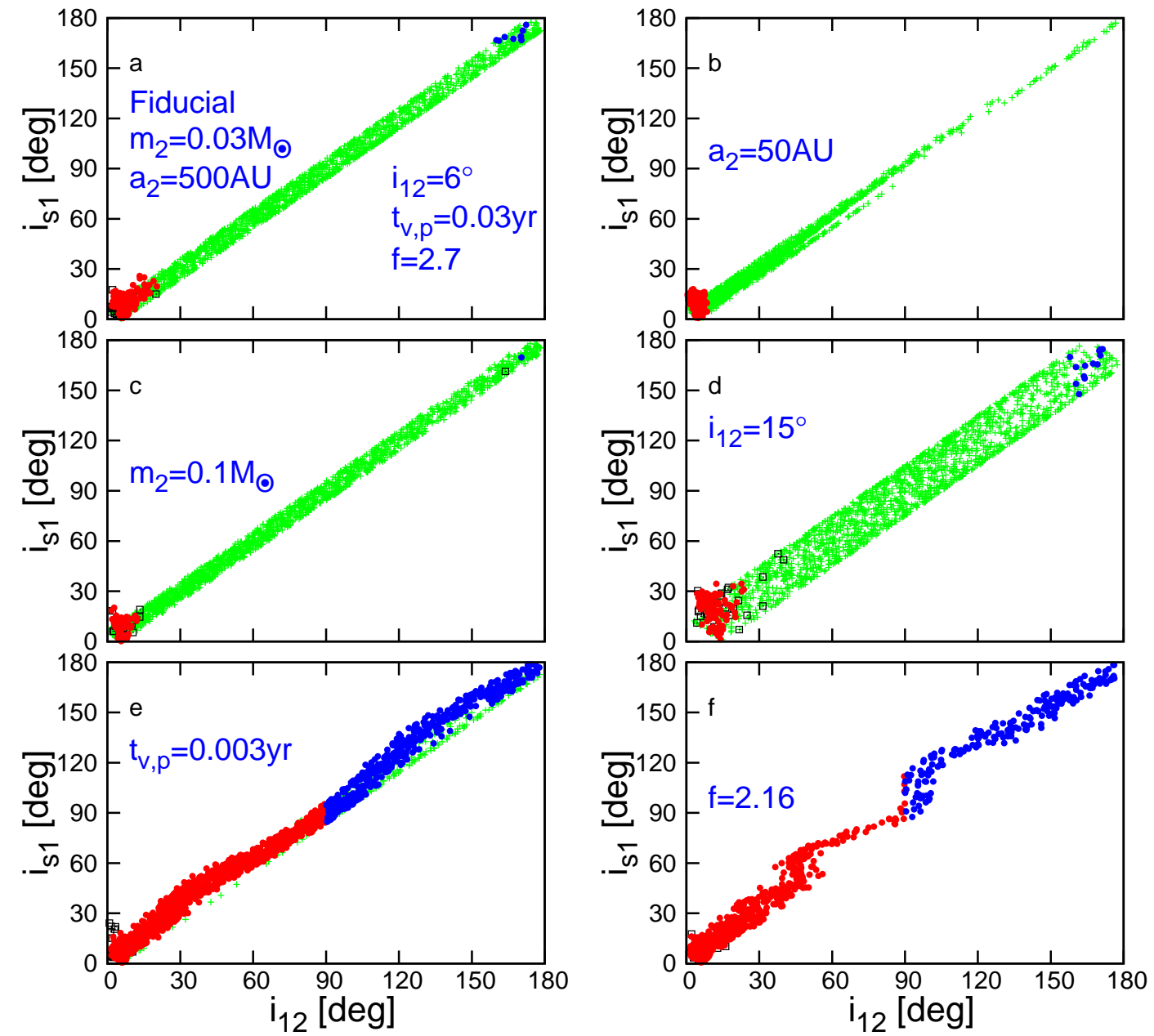

Fig. 12.- Orbital mutual orbital inclination against the spin-orbit angle between the central star and the inner planet. The different colors indicate the different final outcomes of the inner planet; NM (black), PHJ (red), RHJ (blue), and TD (green). 

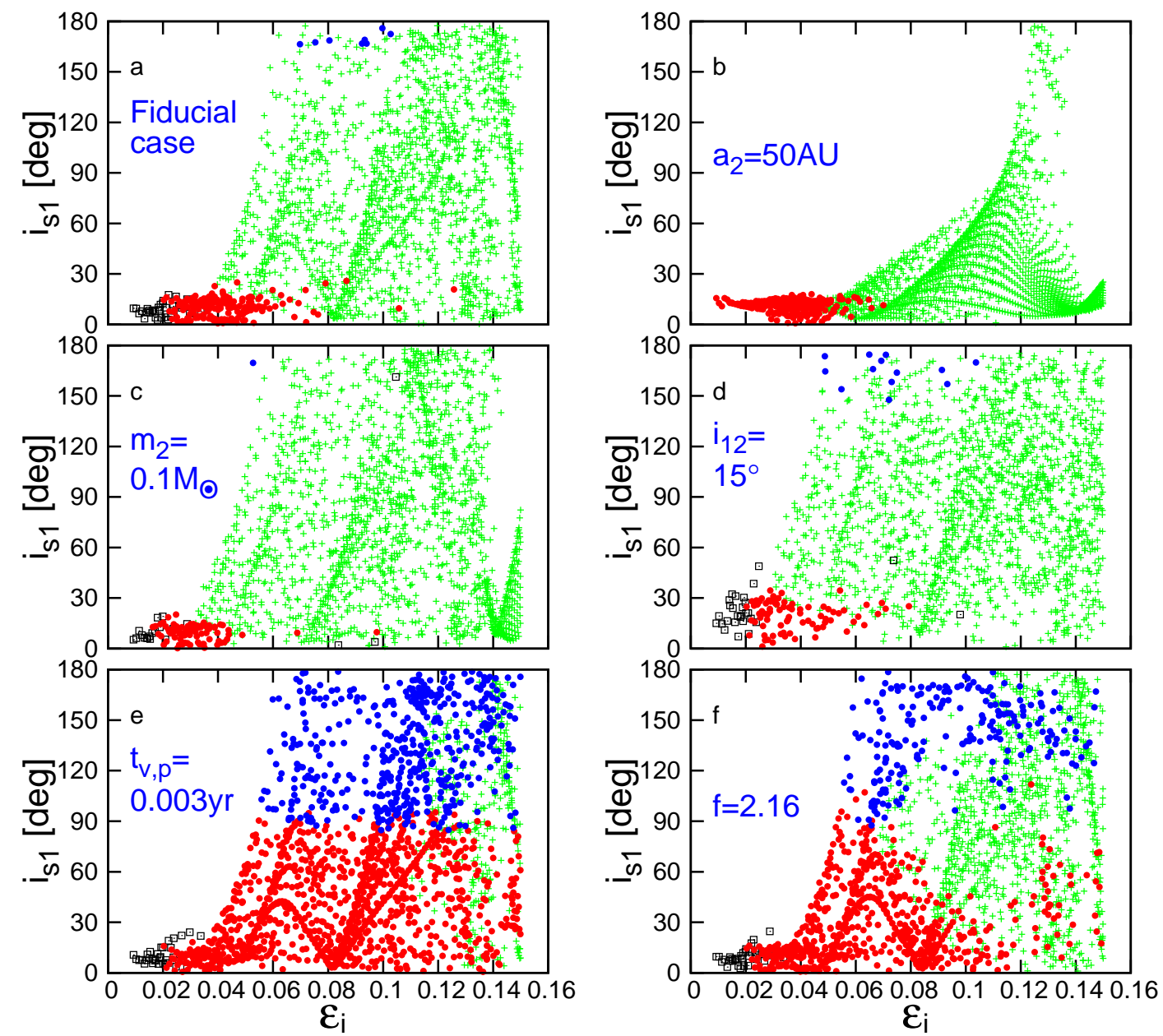

Fig. 13. - Spin-orbit angles $i_{s 1}$ for our models; a: fiducial, b: a50, c: m01, d: i15, e: t00030, and f: f216. The different colors indicate the different final outcomes of the inner planet; NM (black), PHJ (red), RHJ (blue), and TD (green). 


\section{Summary and discussion}

The observation of the Rossiter-McLaughlin effect has revealed a dozen of possible retrograde planets, which already has challenged the conventional theory of planet formation. Although there exists no reliable candidate (yet), the presence of counter-orbiting planets would have an even stronger impact on the formation theory; somewhat conventional planetary migration scenarios including disk-planet interaction, planet-planet scattering, and the Lidov-Kozai migration are successful in producing retrograde planets, but fail to explain the counter-orbiting planets in general.

An interesting and attractive possibility is based on the extreme eccentricity evolution expected for the near-coplanar hierarchical triple system. Indeed Li et al. (2014) and Petrovich (2015b) derived an analytical condition for the orbital flip of the inner planet, which holds for the massless limit of the inner planet under the quadrupole and octupole gravitational potentials of the outer perturber but neglecting the short-range forces (GR, star and inner planetary tide, and rotational distortion) .

In the present paper, we have performed a series of systematic simulations for the sub-stellar outer perturber case, including the short-range forces and examined in detail the condition for the orbital flip in a more realistic situation.

Our main findings are summarized as follows;

1) Most of the near-coplanar hierarchical triple systems that satisfy the analytical flip condition do not produce counter-orbiting planets. Instead, the inner planets in those systems are tidally disrupted. A small fraction of the systems end up with the prograde Hot Jupiters, and very few retrograde Hot Jupiters are produced. Systems that do not satisfy the analytical flip condition do not exhibit any significant migration of the inner planet.

2) The break-down of the the analytical flip condition is due to the short-range forces, which suppresses the extreme eccentricity evolution of the inner planet that is required for the orbital flip.

3) The results are almost independent of the model parameters, and thus fairly generic unless unrealistically strong tidal effect is assumed.

4) The mutual orbital inclination angle between the inner planet and outer perturber, and the spin-orbit angle between the central star and the inner planet are almost the same. Their distribution for the survived Hot Jupiters is bimodal; $\sim 0^{\circ}-20^{\circ}$ for prograde, and $\sim 160^{\circ}-180^{\circ}$ for retrograde planets, and virtually nothing in-between.

Our simulation runs span the parameter space that satisfy the analytical flip condition, and more importantly uniformly sample the $\epsilon_{1, i}-e_{1, i}$ plane without assuming any prior distribution for their realistic values. Therefore predicted statistics for the fate of the inner planet under such configurations are significantly biased. Having emphasized such warnings, however, it might

be instructive to present some statistics simply illustrating the difficulty of forming the counterorbiting planets in the near-coplanar hierarchical triple system. 
Figure 14 plots the fraction of four different final outcomes of the inner planet; NM (nonmigrating planet) in black, PHJ (prograde Hot Jupiter)) in red, RHJ (retrograde Hot Jupiter) in blue, and TD (tidally disrupted planet) in green. The left panel corresponds to a number fraction of each fate simply from the numbers out of 1800 runs for each model summarized in Table 1 . The right panel is computed from their sub-sample with $10 \mathrm{AU}<a_{1, i}<30 \mathrm{AU}$ so as to sample the $a_{1, i}-$ $e_{1, i}$ plane assuming the eccentric inner gas giant planets orbiting at reasonable distances from the central star just for the comparison purpose.
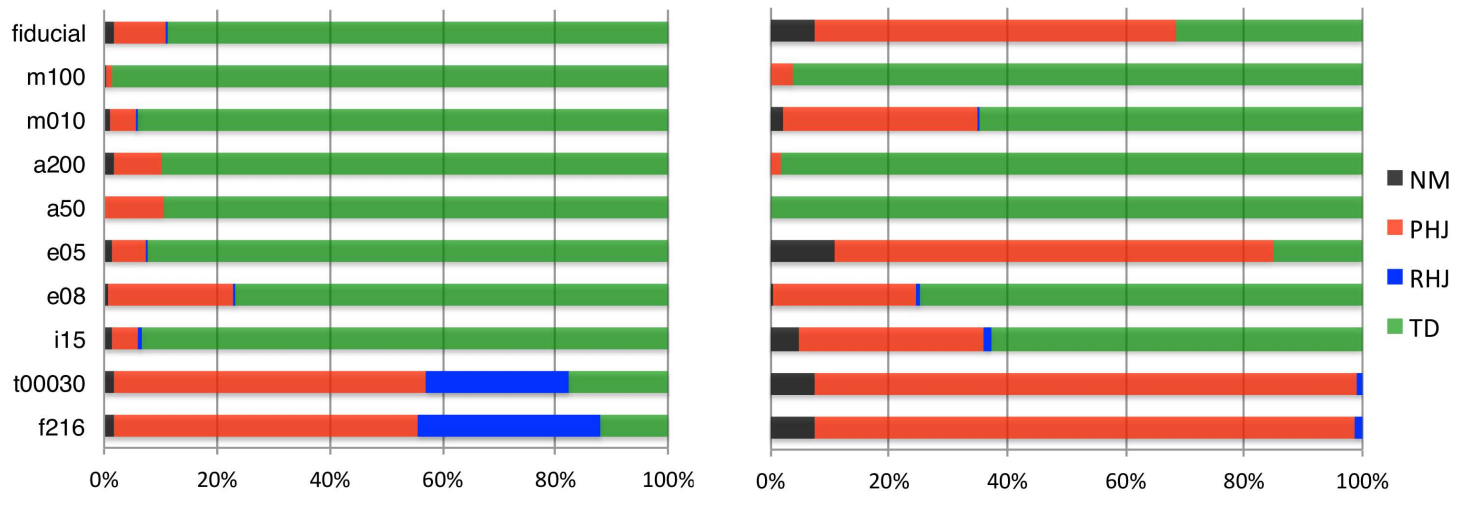

Fig. 14. - The fraction of the final outcome of the inner planets. Left panel: All simulation runs. Right panel: $10 \mathrm{AU}<\mathrm{a}_{1, \mathrm{i}}<30 \mathrm{AU}$.

In any case, our basic conclusion remains the same even if the statistics shown here just for example may be highly biased; it is very difficult to produce the retrograde planet in the present scenario, while some fraction of prograde Hot Jupiter might have formed through this channel. This implies that the formation of counter-orbiting planets imposes an even more serious challenge for the theory. Instead, it could be simply the case that counter-orbiting planet candidates with the projected spin-orbit angle $\lambda \approx 180^{\circ}$ are mildly misaligned with their true spin-orbit angles $\psi$ being much less than $180^{\circ}$ as suggested for HAT-P-7b (Benomar et al. 2014). In this respect, future observational search for the counter-orbiting planets combined with the Rossiter-McLaughlin effect and asteroseismology continues to be important, and hopefully will bring an exciting puzzle for planet formation.

Finally we note that the presence of numerous tidally disrupted planets is not specific to the near-coplanar hierarchical triple systems, but a fairly generic outcome in planetary migration models and in spin-orbit realignment models (Lai 2012; Rogers \& Lin 2013; Xue et al. 2014; Li \& Winn 2015). Thus it is of vital importance to look for possible signatures of such tidal disruption events observationally. Indeed recent studies for the determination of the orbital decay rate (Jiang 2015) and for the unsual photometric signals in KIC 8462852 (Boyajian et al. 2015; Bodman \& Quillen 2015), for instance, are closely related to such an important direction. 
We thank Shoya Kamiaka and Kento Masuda for useful discussions. We are also grateful to an anonymous referee for several important suggestions that improved our earlier manuscript. This research is supported by the Grant-in Aid for Scientific Research by Japan Society of Promotion of Science No. 24340035.

\section{Appendix A. Basic equations for secular evolution}

Just for self-containedness, we write the secular equations of motion used in the present paper for a hierarchical triple system. We consider gravitational interaction up to the octupole expansion of the outer body as described by Liu et al. (2015). In addition, we include the general relativistic correction, the spin effect of the central star and the inner planet, and tidal effect following Correia et al. (2011). In addition, we incorporate the damping of the stellar spin due to magnetic braking following Barker \& Ogilvie (2009).

The subscripts 0,1 , and 2 distinguish the quantities for the central star, the inner planet and the outer perturber, respectively. The mass and radius of those objects are denoted by $m$ and $R$. The spin rate $\omega_{i}$ and gravity coefficients $J_{2_{i}}$ for the star $(i=0)$ and inner planet $(i=1)$ are written as

$$
J_{2_{i}}=k_{2_{i}} \frac{\omega_{i}^{2} R_{i}^{3}}{3 G m_{i}}
$$

where $k_{2_{i}}$ is the second Love number that characterizes the deformation property of each body.

All the equations are written in Jacobi coordinates with $\mathbf{r}_{1}$ being the relative position from $m_{0}$ to $m_{1}$, and $a$ and $e$ are semi-major axis and eccentricity, respectively. Then, the evolution of spin and orbit can be tracked in the octupole approximation by three parameters; spin angular momentum:

$$
\mathbf{L}_{i}=C_{i} \omega_{i} \hat{\mathbf{s}}_{i}
$$

where $\hat{\mathbf{s}}_{i}$ is the unit vector of $\hat{\mathbf{L}}_{i}$ and $C_{i}$ is the principal moment of inertia, the orbital angular momentum:

$$
\mathbf{G}_{i}=\beta_{i} \sqrt{\mu_{i} a_{i}\left(1-e_{i}^{2}\right)} \hat{\mathbf{k}}_{i}
$$

where $\hat{\mathbf{k}}_{i}$ is the unit vector of $\hat{\mathbf{G}}_{i}$ with $\beta_{1}=m_{0} m_{1} /\left(m_{0}+m_{1}\right), \beta_{2}=\left(m_{0}+m_{1}\right) m_{2} /\left(m_{0}+m_{1}+m_{2}\right)$, $\mu_{1}=G\left(m_{0}+m_{1}\right)$ and $\mu_{2}=G\left(m_{0}+m_{1}+m_{2}\right)$, and finally the Lenz vector:

$$
\mathbf{e}_{1}=\frac{\left(\dot{\mathbf{r}}_{1} \times \mathbf{G}_{1}\right)}{\beta_{1} \mu_{1}}-\frac{\mathbf{r}_{1}}{r_{1}}
$$

We define direction angles as

$$
\cos \theta_{i}=\hat{\mathbf{s}}_{i} \cdot \hat{\mathbf{k}}_{1}, \quad \cos \epsilon_{i}=\hat{\mathbf{s}}_{i} \cdot \hat{\mathbf{k}}_{2}, \quad \cos i_{12}=\hat{\mathbf{k}}_{1} \cdot \hat{\mathbf{k}}_{2},
$$

where $\theta_{i}$ is the angle between the spin of the $i$-th body (in the main text we use $i_{s 1}$ to denote $\theta_{0}$ ), $\hat{\mathbf{s}}_{i}$ and inner orbit, $\hat{\mathbf{k}}_{1}, \epsilon_{i}$ is the angle between the spin of the $i$-th body, $\hat{\mathbf{s}}_{i}$, and outer orbit $\hat{\mathbf{k}}_{2}$, and $i_{12}$ is inclination between two orbits. 
Averaging the equations of motion over the mean anomalies of the inner and outer bodies, we obtain the following equations for the conservative motion:

$$
\begin{aligned}
\dot{\mathbf{G}}_{1}= & -\gamma\left(1-e_{1}^{2}\right) \cos i_{12} \hat{\mathbf{k}}_{2} \times \hat{\mathbf{k}}_{1}+5 \gamma\left(\mathbf{e}_{1} \cdot \hat{\mathbf{k}}_{2}\right) \hat{\mathbf{k}}_{2} \times \mathbf{e}_{1} \\
& -\frac{25}{16} \epsilon_{\text {oct }} \gamma\left\{\left[2\left(1-e_{1}^{2}\right)\left[\left(\mathbf{e}_{1} \cdot \hat{\mathbf{e}}_{2}\right) \cos i_{12}+\left(\mathbf{e}_{1} \cdot \hat{\mathbf{k}}_{2}\right)\left(\hat{\mathbf{k}}_{1} \cdot \hat{\mathbf{e}}_{2}\right)\right] \hat{\mathbf{k}}_{1}\right.\right. \\
& \left.+2\left[\left(1-e_{1}^{2}\right)\left(\hat{\mathbf{k}}_{1} \cdot \hat{\mathbf{e}}_{2}\right) \cos i_{12}-7\left(\mathbf{e}_{1} \cdot \hat{\mathbf{k}}_{2}\right)\left(\mathbf{e}_{1} \cdot \hat{\mathbf{k}}_{2}\right)\right] \mathbf{e}_{1}\right] \times \hat{\mathbf{k}}_{2} \\
& +\left[2\left(1-e_{1}^{2}\right)\left(\mathbf{e}_{1} \cdot \hat{\mathbf{k}}_{2}\right) \cos i_{12} \hat{\mathbf{k}}_{1}+\left[\frac{8}{5} \mathbf{e}_{1}^{2}-\frac{1}{5}\right.\right. \\
& \left.\left.\left.-7\left(\mathbf{e}_{1} \cdot \hat{\mathbf{k}}_{2}\right)^{2}+\left(1-e_{1}^{2}\right) \cos ^{2} i_{12}\right] \mathbf{e}_{1}\right] \times \hat{\mathbf{e}_{2}}\right\} \\
& -\sum_{i} \alpha_{1 i} \cos \theta_{i} \hat{\mathbf{s}}_{i} \times \hat{\mathbf{k}}_{1}, \\
\dot{\mathbf{G}}_{2}= & -\gamma\left(1-e_{1}^{2}\right) \cos i_{12} \hat{\mathbf{k}}_{1} \times \hat{\mathbf{k}}_{2}+5 \gamma\left(\mathbf{e}_{1} \cdot \hat{\mathbf{k}}_{2}\right) \mathbf{e}_{1} \times \hat{\mathbf{k}}_{2} \\
& +\frac{25}{16} \epsilon_{\mathrm{oct}} \gamma\left\{\left[2\left(1-e_{1}^{2}\right)\left[\left(\mathbf{e}_{1} \cdot \hat{\mathbf{e}}_{2}\right) \cos i_{12}+\left(\mathbf{e}_{1} \cdot \hat{\mathbf{k}}_{2}\right)\left(\hat{\mathbf{k}}_{1} \cdot \hat{\mathbf{e}}_{2}\right)\right] \hat{\mathbf{k}}_{1}\right.\right. \\
& \left.+2\left[\left(1-e_{1}^{2}\right)\left(\hat{\mathbf{k}}_{1} \cdot \hat{\mathbf{e}}_{2}\right) \cos i_{12}-7\left(\mathbf{e}_{1} \cdot \hat{\mathbf{k}}_{2}\right)\left(\mathbf{e}_{1} \cdot \hat{\mathbf{k}}_{2}\right)\right] \mathbf{e}_{1}\right] \times \hat{\mathbf{k}}_{2} \\
& +\left[2\left(1-e_{1}^{2}\right)\left(\mathbf{e}_{1} \cdot \hat{\mathbf{k}}_{2}\right) \cos i_{12} \hat{\mathbf{k}}_{1}+\left[\frac{8}{5} \mathbf{e}_{1}^{2}-\frac{1}{5}\right.\right. \\
& \left.\left.\left.-7\left(\mathbf{e}_{1} \cdot \hat{\mathbf{k}}_{2}\right)^{2}+\left(1-e_{1}^{2}\right) \cos { }^{2} i_{12}\right] \mathbf{e}_{1}\right] \times \hat{\mathbf{e}}_{2}\right\} \\
& -\sum_{i} \cos \epsilon_{i} \hat{\mathbf{s}}_{i} \times \hat{\mathbf{k}}_{2}, \\
& {[2}
\end{aligned}
$$

$$
\begin{aligned}
\dot{\mathbf{e}}_{1}= & -\frac{\gamma\left(1-e_{1}^{2}\right)}{\left\|\mathbf{G}_{1}\right\|}\left[\cos i_{12} \hat{\mathbf{k}}_{2} \times \mathbf{e}_{1}-2 \hat{k}_{1} \times \mathbf{e}_{1}-5\left(\mathbf{e}_{1} \cdot \hat{\mathbf{k}}_{2}\right) \hat{\mathbf{k}}_{2} \times \hat{\mathbf{k}}_{1}\right] \\
& -\frac{25}{16} \epsilon_{\mathrm{oct}} \gamma\left\{2 \sqrt { ( 1 - e _ { 1 } ^ { 2 } ) } \left[\left(\mathbf{e}_{1} \cdot \hat{\mathbf{k}}_{2}\right) \cos i_{12} \mathbf{e}_{1}\right.\right. \\
& \left.+\left[\frac{8}{5} \mathbf{e}_{1}^{2}-\frac{1}{5}-7\left(\mathbf{e}_{1} \cdot \hat{\mathbf{k}}_{2}\right)^{2}+\left(1-e_{1}^{2}\right) \cos ^{2} i_{12}\right] \hat{\mathbf{k}}_{1}\right] \times \hat{\mathbf{e}_{2}} \\
& +2 \sqrt{\left(1-e_{1}^{2}\right)}\left[\left[\left(\mathbf{e}_{1} \cdot \hat{\mathbf{e}}_{2}\right) \cos i_{12}+\left(\mathbf{e}_{1} \cdot \hat{\mathbf{k}}_{2}\right)\left(\hat{\mathbf{k}}_{1} \cdot \hat{\mathbf{e}}_{2}\right)\right] \mathbf{e}_{1}\right. \\
& \left.\left.+\left[\left(\hat{\mathbf{k}}_{1} \cdot \hat{\mathbf{e}}_{2}\right) \cos i_{12}-7\left(\mathbf{e}_{1} \cdot \hat{\mathbf{k}}_{2}\right)\left(\mathbf{e}_{1} \cdot \hat{\mathbf{e}}_{2}\right)\right] \hat{\mathbf{k}}_{1}\right] \times \hat{\mathbf{k}}_{2}+\frac{16}{5}\left(\mathbf{e}_{1} \cdot \hat{\mathbf{e}}_{2}\right) \sqrt{\left(1-e_{1}^{2}\right)} \mathbf{k}_{1} \times \mathbf{e}_{1}\right\} \\
& -\sum_{i} \frac{\alpha_{1 i}}{\left\|\mathbf{G}_{1}\right\|}\left[\cos \theta_{i} \hat{\mathbf{s}}_{i} \times \mathbf{e}_{1}+\frac{1}{2}\left(1-5 \cos ^{2} \theta_{i}\right) \hat{\mathbf{k}}_{1} \times \mathbf{e}_{1}\right],
\end{aligned}
$$




$$
\begin{aligned}
& \dot{\mathbf{e}}_{2}= \frac{\gamma}{\sqrt{1-e_{2}^{2}}} \frac{\beta_{1} \sqrt{\mu_{1} a_{1}}}{\beta_{2} \sqrt{\mu_{2} a_{2}}}\left[\left(1-e_{1}^{2}\right) \cos i_{12} \mathbf{e}_{2} \times \hat{\mathbf{k}}_{1}-5\left(\mathbf{e}_{1} \cdot \hat{\mathbf{k}}_{2}\right) \mathbf{e}_{2} \times \mathbf{e}_{1}\right. \\
&\left.-\left[\frac{1}{2}-3 e_{1}^{2}+\frac{25}{2}\left(\mathbf{e}_{1} \cdot \hat{\mathbf{k}}_{2}\right)^{2}-\frac{5}{2}\left(1-e_{1}^{2}\right) \cos ^{2} i_{12}\right] \hat{\mathbf{k}}_{2} \times \mathbf{e}_{2}\right] \\
&-\frac{25}{16} \epsilon_{\mathrm{oct}} \gamma \sqrt{1-e_{2}^{2}} \frac{\beta_{1} \sqrt{\mu_{1} a_{1}}}{\beta_{2} \sqrt{\mu_{2} a_{2}}}\left\{2 ( 1 - e _ { 1 } ^ { 2 } ) \left[\left(\mathbf{e}_{1} \cdot \hat{\mathbf{k}}_{2}\right)\left(\hat{\mathbf{k}}_{1} \cdot \mathbf{e}_{2}\right) \hat{\mathbf{e}}_{2}\right.\right. \\
&\left.+\frac{1-e_{2}^{2}}{e_{2}}\left[\frac{8}{5} \mathbf{e}_{1}^{2}-\frac{1}{5}-7\left(\mathbf{e}_{1} \cdot \hat{\mathbf{k}}_{2}\right)^{2}+\left(1-e_{1}^{2}\right) \cos ^{2} i_{12}\right] \hat{\mathbf{k}}_{2}\right] \times \mathbf{e}_{1} \\
&-\left[2\left(\frac{1}{5}-\frac{8}{5} \mathbf{e}_{1}^{2}\right)\left(\mathbf{e}_{1} \cdot \hat{\mathbf{e}}_{2}\right) \mathbf{e}_{2}+14\left(1-e_{1}^{2}\right)\left(\mathbf{e}_{1} \cdot \hat{\mathbf{k}}_{2}\right)\left(\hat{\mathbf{k}}_{1} \cdot \hat{\mathbf{e}}_{2}\right)\left(\hat{\mathbf{k}}_{1} \cdot \hat{\mathbf{k}}_{2}\right) \mathbf{e}_{2}\right. \\
&\left.\left.\left.+7\left(\mathbf{e}_{1} \cdot \hat{\mathbf{e}}_{2}\right)\left[\frac{8}{5} \mathbf{e}_{1}^{2}-\frac{1}{5}-7\left(\mathbf{e}_{1} \cdot \hat{\mathbf{k}}_{2}\right)^{2}+\left(1-e_{1}^{2}\right) \cos ^{2} i_{12}\right] \mathbf{e}_{2}\right] \times \hat{\mathbf{k}}_{2}\right)\right\} \\
& \dot{\mathbf{L}}_{i}=\alpha_{1 i} \cos \theta_{i} \hat{\mathbf{s}}_{i} \times \hat{\mathbf{k}}_{1}+\alpha_{2 i} \cos \epsilon_{i} \hat{\mathbf{s}}_{i} \times \hat{\mathbf{k}}_{2} .
\end{aligned}
$$

where

$$
\begin{aligned}
\epsilon_{\mathrm{oct}} & =\frac{m_{0}-m_{1}}{m_{0}+m_{1}} \frac{a_{1}}{a_{2}} \frac{e_{2}}{1-e_{2}^{2}}, \\
\alpha_{1 i} & =\frac{3 G m_{0} m_{1} J_{2 i} R_{i}^{2}}{2 a_{1}^{3}\left(1-e_{1}^{2}\right)^{3 / 2}}, \\
\alpha_{2 i} & =\frac{3 G m_{2} m_{i} J_{2 i} R_{i}^{2}}{2 a_{2}^{3}\left(1-e_{2}^{2}\right)^{3 / 2}}, \\
\gamma & =\frac{3 G m_{2} \beta_{1} a_{1}^{2}}{4 a_{2}^{3}\left(1-e_{2}^{2}\right)^{3 / 2}} .
\end{aligned}
$$

In the above expressions, the parameter $\epsilon_{\text {oct }}$ quantifies the importance of the octupole term relative to the quadrupole term.

The magnetic braking as a spin-down process of the central star is modelled as

$$
\dot{\mathbf{L}}_{0, \mathrm{mb}}=-\alpha_{\mathrm{mb}} C_{0} \omega_{0}^{3} \hat{\mathbf{s}}_{0}
$$

where the spin-down rate $\alpha_{\mathrm{mb}}$ is set to be $1.66 \times 10^{-13}$ yr according to Barker \& Ogilvie (2009). Incidentally the same magnetic braking effect was incorporated in Xue et al. (2014) although it was not noted explicitly.

The correction due to general relativity induces the precession of the pericenter:

$$
\dot{\mathbf{e}}_{1, \mathrm{GR}}=\frac{3 \mu_{1} n_{1}}{c^{2} a_{1}\left(1-e_{1}^{2}\right)} \hat{\mathbf{k}}_{1} \times \mathbf{e}_{1}
$$

where $c$ is light speed, and $n_{1}$ is mean motion of the inner orbit. 
For the tidal effect, we adopt the equilibrium tidal model with constant delay time $\Delta t_{i}$ (Mignard 1979). Similarly, the averaged equations are

$$
\begin{gathered}
\dot{\mathbf{G}}_{2, \text { tide }}=0, \quad \dot{\mathbf{G}}_{1, \text { tide }}=-\dot{\mathbf{L}}_{0}-\dot{\mathbf{L}}_{1}, \\
\dot{\mathbf{e}}_{1, \text { tide }}=\sum_{i} \frac{15}{2} k_{2_{i}} n_{1}\left(\frac{m_{(1-i)}}{m_{i}}\right)\left(\frac{R_{i}}{a_{1}}\right)^{5} f_{4}\left(e_{1}\right) \hat{\mathbf{k}}_{1} \times \mathbf{e}_{1} \\
-\sum_{i} \frac{K_{i}}{\beta_{1} a_{1}^{2}}\left[f_{4}\left(e_{1}\right) \frac{\omega_{i}}{2 n_{1}}\left(\mathbf{e}_{1} \cdot \hat{\mathbf{s}}_{i}\right) \hat{\mathbf{k}}_{1}-\left(\frac{11}{2} f_{4}\left(e_{1}\right) \cos \theta_{i} \frac{\omega_{i}}{n_{1}}-9 f_{5}\left(e_{1}\right)\right) \mathbf{e}_{1}\right], \\
\dot{\mathbf{L}}_{i, \text { tide }}=K_{i} n_{1}\left[f_{4}\left(e_{1}\right) \sqrt{1-e_{1}^{2}} \frac{\omega_{i}}{2 n_{1}}\left(\hat{\mathbf{s}}_{i}-\cos \theta_{i} \hat{\mathbf{k}}_{1}\right)\right. \\
\left.-f_{1}\left(e_{1}\right) \frac{\omega_{i}}{n_{1}} \hat{\mathbf{s}}_{i}+f_{2}\left(e_{1}\right) \hat{\mathbf{k}}_{1}+\frac{\left(\mathbf{e}_{1} \cdot \hat{\mathbf{s}}_{i}\right)\left(6+e_{1}^{2}\right)}{4\left(1-e_{1}^{2}\right)^{9 / 2}} \frac{\omega_{i}}{n_{1}} \mathbf{e}_{1}\right] .
\end{gathered}
$$

where

$$
\begin{gathered}
K_{i}=\Delta t_{i} \frac{3 k_{2_{i}} G m_{(1-i)}^{2} R_{i}^{5}}{a_{1}^{6}}, \\
f_{1}(e)=\frac{1+3 e^{2}+3 e^{4} / 8}{\left(1-e^{2}\right)^{9 / 2}} \\
f_{2}(e)=\frac{1+15 e^{2} / 2+45 e^{4} / 8+5 e^{6} / 16}{\left(1-e^{2}\right)^{6}}, \\
f_{3}(e)=\frac{1+31 e^{2} / 2+255 e^{4} / 8+185 e^{6} / 16+25 e^{8} / 64}{\left(1-e^{2}\right)^{15 / 2}}, \\
f_{4}(e)=\frac{1+3 e^{2} / 2+e^{4} / 8}{\left(1-e^{2}\right)^{5}}, \\
f_{5}(e)=\frac{1+15 e^{2} / 4+15 e^{4} / 8+5 e^{6} / 64}{\left(1-e^{2}\right)^{13 / 2}} .
\end{gathered}
$$

\section{Appendix B. Short-range force effects: Precession rate on $\hat{\mathrm{e}}_{1}$}

The three main short-range forces (GR, planetary tide and rotational distortion) modify $\hat{\mathbf{e}}_{1}$, and induce an additional precession of $\hat{\mathbf{e}}_{1}$ around $\hat{\mathbf{k}}_{1}$ :

$$
\dot{\mathbf{e}}_{1}=\omega_{\mathrm{pre}} \hat{\mathbf{k}}_{1} \times \mathbf{e}_{1} \text {. }
$$

The precession rate, $\omega_{\text {pre }}$, for the three main short-range forces can be read off from the 
evolution equations in Appendix A in a straightfoward manner as

$$
\begin{aligned}
\omega_{\mathrm{GR}} & =\frac{3 \mu_{1} n_{1}}{c^{2} a_{1}\left(1-e_{1}^{2}\right)} \propto \frac{1}{a_{1}^{5 / 2}\left(1-e_{1}^{2}\right)} \\
\omega_{\mathrm{PT}} & =\frac{15}{2} k_{2_{1}} n_{1}\left(\frac{m_{0}}{m_{1}}\right)\left(\frac{R_{1}}{a_{1}}\right)^{5} f_{4}\left(e_{1}\right) \propto \frac{1}{a_{1}^{13 / 2}\left(1-e_{1}^{2}\right)^{5}}, \\
\omega_{\mathrm{PRD}} & =\frac{\alpha_{11}}{\left\|\mathbf{G}_{1}\right\|} \frac{1}{2}\left(1-5 \cos ^{2} \theta_{1}\right) \propto \frac{1}{a_{1}^{7 / 2}\left(1-e_{1}^{2}\right)^{2}},
\end{aligned}
$$

where $\omega_{\mathrm{GR}}, \omega_{\mathrm{PT}}$, and $\omega_{\mathrm{PRD}}$ are the precession rate induced by GR, planetary tide, and planetary rotational distortion, respectively.

Note that the above expressions are consistent with those of Liu et al. (2015) if the tidal Love number is set to be twice of the deformation love number $k_{2_{i}}$, and the spin and orbit of the inner planet are aligned $\left(\theta_{1}=0^{\circ}\right)$.

\section{Appendix C. Effect of the spin rotation period of the inner planet}

Throughout the present analysis, we have adopted 10 days as the spin rotation period of the inner planet. If one considers Jupiter as a typical planet, 10 hours, instead of 10 days, may be more relevant. Therefore we repeat our fiducial run using the 10 hour period while keeping all the other parameters unchanged. Figure 15 shows the result, which is basically identical with Figure 2. Just for more quantitative comparison, we show the branching ratios of the final outcomes; PHJ 8.5\%, RHJ $0.4 \%$, NM 2.1\%, and TD $89.0 \%$. Thus we conclude that the final result is very insensitive to the choice of the planetary spin period in this range.

\section{REFERENCES}

Albrecht, S., Winn, J. N., Johnson, J. A., et al. 2012, ApJ, 757, 18

Alibert Y., Mordasini C., Benz W., Winisdoerffer C., 2005, A\&A, 434, 343

Anderson K. R., Storch N. I., Lai D., 2016, MNRAS, 456, 3671

Barker, A. J., \& Ogilvie, G. I. 2009, MNRAS, 395, 2268

Bate, M. R., Lodato, G., \& Pringle, J. E. 2010, MNRAS, 401, 1505

Batygin, K. 2013, Natur, 491, 418

Batygin, K., Bodenheimer P. H., Laughlin G. P. 2015, arXiv:1511.09157

Beauge C., \& Nesvorny D., 2012, AJ 751, 119 


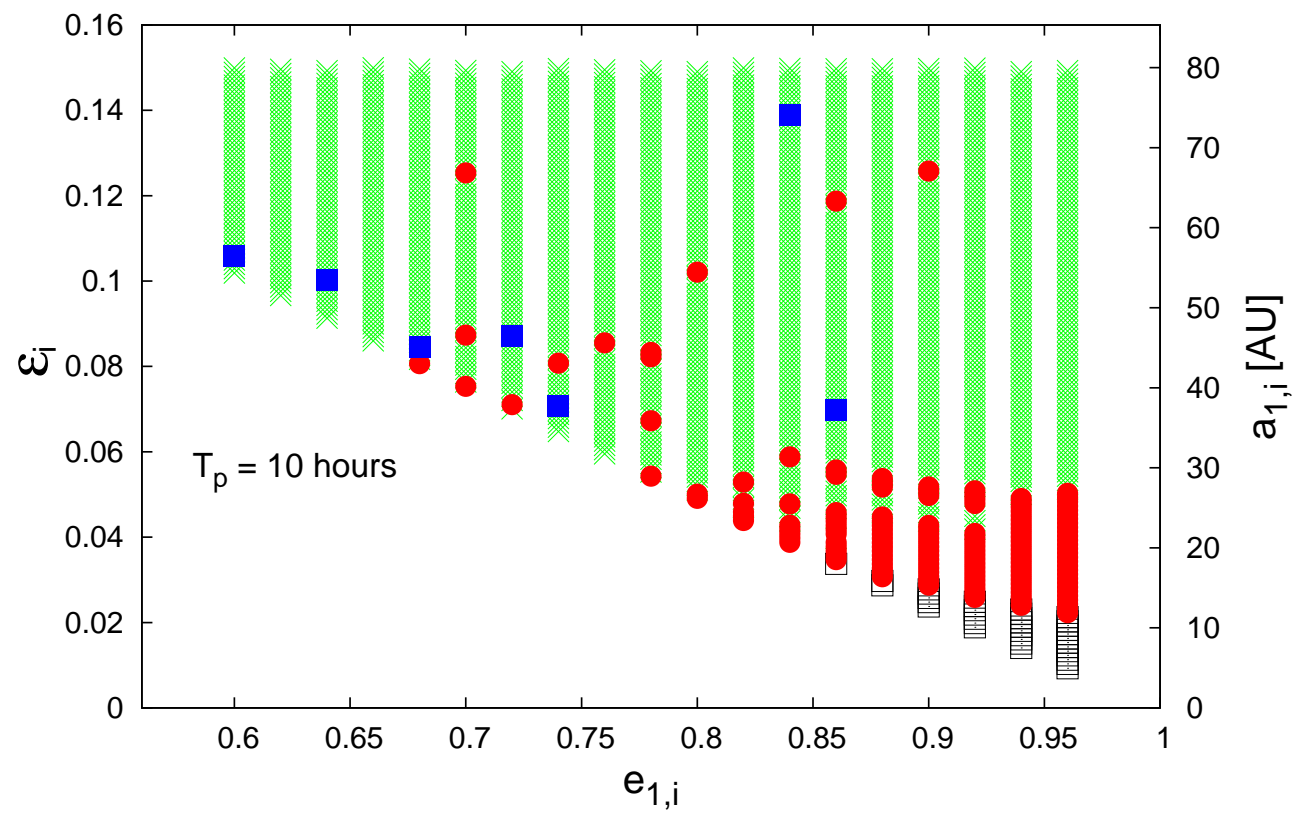

Fig. 15. - Same as Figure 2 but with $T_{p}=10$ hours as the planetary spin rotation period.

Benomar, O., Masuda, K., Shibahashi, H., \& Suto, Y. 2014, PASJ, 66, 94

Bodman, E.H., \& Quillen, A. 2015, arXiv:1511.08821

Boley A.C., Granados C.B., Gladman B 2015, arXiv:1510.04276

Boyajian, T.S., et al. 2015, arXiv:1509.03622

Campante, T. L., ph.D.thesis, arXiv:1405.3145

Christensen-Dalsgaard, J. 2014, Lecture Notes on "Stellar Oscillations", fifth edition, http://astro.phys.au.dk/ jcd/oscilnotes/

Correia, A. C. M., Laskar, J., Farago, F., \& Boue, G. 2011, Celest. Mech. Dyn. Astron., 111, 105

Faber, J. A., Rasio, F. A., \& Willems, B. 2005, Icarus, 175, 248

Fabrycky,D. \& Tremaine, S. 2007, ApJ, 669, 1298

Foucart, F., \& Lai, D. 2011, MNRAS, 412, 2799

Gizon, L., \& Solanki, S.K. 2003, ApJ589,1009

Guillochon, J., Ramirez-Ruiz, E., \& Lin, D. 2011, ApJ, 732, 74

Huber, D., et al. 2013, Science, 342, 331 
Jiang, I.G., et al. 2015, arXiv:1511.00768

Kozai, Y. 1962, AJ, 67, 591

Lai, D. 2012, MNRAS, 423, 486

Lai, D. 2014, MNRAS, 440, 3532

Li, G., Naoz, S., Kocsis, B., \& Loeb, A. 2014, ApJ, 785, 116

Li, G., \& Winn, J. N. 2015, ApJ, in press, arXiv:1511.05570

Lin, D. N. C., Bodenheimer, P., \& Richardson, D. 1996, Nature, 380, 606

Lidov, M. L. 1962, P\&SS, 9, 719

Liu, B., Munoz, D. J., \& Lai, D. 2015, MNRAS, 447, 747

McLaughlin, D. B. 1924, ApJ, 60, 22

Mignard, F., Moon Planets 1979, 20, 301-315

Murray, C.D., \& Dermott, S.F. 1999, Solar System Dynamics (Cambridge Univ. Press; Cambridge, New York)

Nagasawa, M., Ida, S., \& Bessho, T. 2008, ApJ, 678, 498

Nagasawa, M., \& Ida, S. 2011, ApJ, 742, 72

Naoz, S., Farr, W. M., Lithwick, Y., Rasio, F. A., \& Teyssandier, J. 2011, Nature, 473, 187

Naoz, S., Farr, W. M., \& Rasio, F.A., 2012, ApJ, 754, L36

Ohta, Y., Taruya, A., \& Suto Y. 2005, ApJ, 622, 1118

Petrovich C., 2015a, ApJ, 799, 27

Petrovich C., 2015b, ApJ, 805, 75

Queloz, D., Eggenberger, A., Mayor, M., Perrier, C., Beuzit, J. L., Naef, D., Sivan, J. P., \& Udry, S. 2000, A\&A, 359, L13

Rasio, F. A., \& Ford, E. B., 1996, Science, 274, 954

Rogers, T.M. , \& Lin, D.N.C. 2013, ApJL, 769:L10

Rossiter, R. A. 1924, ApJ, 60, 15

Socrates, A., Katz, B., \& Dong, S. 2012, arXiv:1209.5724 
Storch, N. I., \& Lai, D. 2014, MNRAS, 438, 2

Teyssandier, J., Naoz, S., Lizarraga, I., \& Rasio, F. 2013, ApJ, 779, 166

Unno, W., Osaki, Y., Ando, H., Saio, H., \& Shibahashi, H. 1989, Nonradial Oscillation of Stars (Tokyo: University of Tokyo Press)

Xue, Y., Suto, Y., Taruya, A., Hirano, T., Fujii, Y., \& Masuda, K. 2014, ApJ, 784, 66

Winn, J. N., Noyes, R.W., Holman, M.J., Charbonneau, D., Ohta, Y., Taruya, A., Suto, Y., Narita, N., Turner, E.L., Johnson, J.A., Marcy, G.W., Butler, R.P., \& Vogt, S.S. 2005, ApJ, 631, 1215

Winn, J. N., Howard, A. W., Johnson, J. A., et al. 2011, AJ, 141, 63

Wu, Y., \& Murray, N. 2003, ApJ, 589, 605

Wu, Y., \& Lithwick, Y. 2011, ApJ, 735,109 